JLAB-THY-03-195

WM-03-109

\title{
Quark-Hadron Duality and Scaling in Reduced QCD
}

\author{
Zoltán Batiz ${ }^{1}$ and Franz Gross ${ }^{2,3}$ \\ ${ }^{1}$ Centro de Física das Interacções Fundamentais (CFIF), \\ Instituto Superior Técnico, P-1049-001 Lisboa, Portugal \\ ${ }^{2}$ Department of Physics, College of William and Mary, Williamsburg, Virginia 23185 \\ ${ }^{3}$ Thomas Jefferson National Accelerator Facility, Newport News, Virginia 23606
}

(Dated: October 31, 2018)

\begin{abstract}
We introduce a generalization of $1+1$ dimensional large $N_{c}$ QCD, which we refer to as "reduced" QCD, or rQCD. In this model gluons and quark momenta live in $1+1$ dimensions only, but the quark spin and all other particles (leptons, and the photon) live in the full $1+3$ dimensions. The bound states of quarks and antiquarks are identical to those originally described by 't Hooft (except that there are new transversely polarized states previously excluded), so the model is exactly soluable. However, significant differences arise when the model is applied to electromagnetic interactions. After reviewing the strongly interacting sector of the theory, we discuss deep inelastic scattering (DIS) in this model, and show that the new states with transverse polarization give the CallanGross relation and remove the pathological features of the original $1+1$ dimensional description. We conclude that rQCD gives a satisfactory description of the phenomenology and provides a deep understanding of both duality and DIS.
\end{abstract}

PACS numbers: 12.39.-x, 11.10St, 13.40.Gp

\section{INTRODUCTION}

In 1974, 't Hooft described the behavior of the large $N_{c}$ limit of QCD in $1+1$ dimensions 1]. He showed that the model confined quarks and was exactly soluable. His work was followed quickly by several papers [2, 3, 4] discussing various aspects of the model. The work by Einhorn [3] discussed the 't Hooft model predictions for deep inelastic scattering (DIS). Einhorn found that the DIS cross section was proportional to the square of the undressed quark masses, and hence would approach zero as the quark masses approach zero. This pathlogical result made it difficult to apply the 't Hooft model to the phenomenology of DIS; clearly someting major was missing. Of course, limiting the physics to $1+1$ dimensions also excludes transverse spin degrees of freedom, so $W_{1}$ was zero and the Callan-Gross relation [5] did not hold.

Since then QCD in $1+1$ diminsions has been studied from a variety of points of view $[6,6,8,9,10]$ and applied recently to the study of duality in heavy meson decays [11, 12, 13]. However, to the best of our knowledge, no one has followed up on Einhorn's study [3] of DIS. 
A few years ago we found that a very simple toy model of scalar and spinor fields in $1+1$ dimension gave nice phenomenological results for DIS [14]. By extending the spinor degrees of freedom to $1+3$ dimensions we were able to recover the Callan-Gross relation. We decided to extend our approach to the 't Hooft model, and learned of the pathology in its description of DIS. It turns out that this pathology can be removed by extending the spinor degrees of freedom to $1+3$ dimensions, just as we did before, and this paper grew out of that study.

This paper is divided into five sections, with seven appendices that include many of the details. Following this brief introduction, Sec. II reviews the 't Hooft model results for the strong sector. Our discussion focuses on (i) a method of treatment of the confining interaction that removes all singularities from the theory and gives finite dressed quark masses, and (ii) the consequence of including transverse degrees of freedom for the spin of the quarks. We calculate the dressed quark mass, and the properties and spectrum of $q \bar{q}$ bound states, and also study the consequences of the completness of the bound states. We show how to construct an off-shell $q \bar{q}$ scattering matrix, and show that the on-shell scattering matrix must be zero, as required for confined particles. Sections III and IV study electromagnetic interactions and deep inelastic scatterng. Here the presence of transverse spin degrees of freedom completly alters the Einhorn discussion, giving us phenomonologically useful results. We conclude with a brief discussion.

In a subsequent paper [15] we plan to present a numerical study of the approach to scaling in the DIS limit.

\section{THE 'T HOOFT MODEL}

\section{A. The Lagrangian and confining interaction}

We propose that the action have the following form

$$
\mathcal{S}=\mathcal{S}_{Q C D}+\mathcal{S}_{Q E D}
$$

where the QCD part of the action (gluons and quark momenta or coordinates) live in $1+1$ dimensions only, but the quark spin and the QED part (leptons and the photon) live in the full $1+3$ dimensions

$$
\begin{aligned}
& \mathcal{S}_{Q C D}=\int d t d z \mathcal{L}_{Q C D}(t, z) \\
& \mathcal{S}_{Q E D}=\int d t d \mathbf{r} \mathcal{L}_{Q E D}(t, \mathbf{r}) .
\end{aligned}
$$

In his original paper, 't Hooft [1] discussed the strong interactions only, and in this section we review the $1+1$ dimensional model of QCD that he presented. The QED part of the action (2.2), and reduced QCD (rQCD), will be discussed in Sec. III]

The QCD Lagrangian density is

$$
\mathcal{L}_{Q C D}(t, z)=-\frac{1}{4} \operatorname{Tr}\left[F^{\mu \nu} F_{\mu \nu}\right]+\sum_{i} \bar{q}_{i}\left(i D_{\mu} \gamma^{\mu}-m_{0 i}\right) q_{i},
$$

where $q_{i}=q_{i}(t, z)$ is the quark field with flavor $i$ and bare mass $m_{0 i}$. The gluon field quantities are

$$
A^{\mu}=\frac{1}{2} A_{a}^{\mu} \lambda_{a}
$$




$$
\begin{aligned}
F_{\mu \nu} & =\partial_{\mu} A_{\nu}-\partial_{\nu} A_{\mu}+i g_{0}\left[A_{\mu}, A_{\nu}\right] \\
D_{\mu} & =\partial_{\mu}+i g_{0} A_{\mu}
\end{aligned}
$$

where $F_{\mu \nu}$ is the gluon field tensor and $A_{a}^{\mu}=A_{a}^{\mu}(t, z)$ are the gluon fields with the Lorentz index $\mu$ and the color index $a$. The gluon fields have components in the 0 and 3 direction only, so the sum over the index $\mu$ is restricted to 0 and 3. The QCD coupling constant is $g_{0}$ and $\lambda^{a}$ are the generators of the $S U\left(N_{c}\right)$ color group, normalized to

$$
\begin{aligned}
& \operatorname{trace}\left[\lambda^{a} \lambda^{b}\right]=2 \delta^{a b} \\
& \sum_{a} \lambda^{a} \lambda^{a}=\frac{2\left(N_{c}^{2}-1\right)}{N_{c}} \mathbb{1},
\end{aligned}
$$

where matrix multiplication of the $\lambda$ matrices is implied. As $N_{c} \rightarrow \infty$, finite results are obtained if $g_{0} \rightarrow 0$ as

$$
g_{0}=\frac{g}{\sqrt{2 N_{c}}} .
$$

where the effective coupling $g$ is a constant.

While the space-time coordinates, momenta, and Lorentz vector sums in Eq. (2.3) are restricted to $1+1$ dimension, we assume that the gamma matrices have the usual $4 \times 4$ Dirac structure. The quark fields are therefore a direct product of four Dirac dimensions and $N_{c}$ color dimensions.

Note that the action (2.2) is Lorentz invariant under the subgroup $G_{3}$ of Lorentz transformations that leave the $x y$ plane invariant. Specifically, the group is generated by the hamiltoninian $\mathcal{H}$, and the boost $\mathcal{K}_{3}$, momentum $\mathcal{P}_{3}$, and angular momentum $\mathcal{J}_{3}$, operators that generate boosts and translations along the $z$ axis, and rotations about the $z$ axis. Four of the six commutation relations between these generators are zero, and the other two close

$$
\left[\mathcal{K}_{3}, \mathcal{P}_{3}\right]=-i \mathcal{H} \quad\left[\mathcal{K}_{3}, \mathcal{H}\right]=-i \mathcal{P}_{3}
$$

guaranteeing that $G_{3}$ is a group.

Following 't Hooft [1] we introduce light cone variables:

$$
\begin{aligned}
& b_{+}=\frac{1}{\sqrt{2}}\left(b^{0}+b^{3}\right) \\
& b_{-}=\frac{1}{\sqrt{2}}\left(b^{0}-b^{3}\right) \\
& \mathbf{b}_{\perp}=\left\{b_{1}, b_{2}\right\}
\end{aligned}
$$

for any arbitrary vector $b$ (in the QCD sector, the only perpendicular components come from matrix elements of $\gamma_{\perp}$ ). Note that the scalar product of any two vectors $a$ and $b$ is

$$
a_{\mu} b^{\mu}=a \cdot b=a_{+} b_{-}+a_{-} b_{+}-\mathbf{a}_{\perp} \cdot \mathbf{b}_{\perp}
$$

The derivatives are defined

$$
\begin{aligned}
& \partial_{-}=\frac{\partial}{\partial x_{+}}=\frac{1}{\sqrt{2}}\left(\partial^{0}-\partial^{3}\right)=\frac{1}{\sqrt{2}}\left(\frac{\partial}{\partial x^{0}}+\frac{\partial}{\partial x^{3}}\right) \\
& \partial_{+}=\frac{\partial}{\partial x_{+}}=\frac{1}{\sqrt{2}}\left(\partial^{0}+\partial^{3}\right)=\frac{1}{\sqrt{2}}\left(\frac{\partial}{\partial x^{0}}-\frac{\partial}{\partial x^{3}}\right)
\end{aligned}
$$


so that the divergence of a two-vector is

$$
\partial_{0} b^{0}+\partial_{3} b^{3}=\partial_{-} b_{+}+\partial_{+} b_{-} .
$$

In the same way, we can define the,+- , and $\perp$ components of the $\gamma$ matrices. The anticommutation relations are all zero except for

$$
\begin{aligned}
& \left\{\gamma_{+}, \gamma_{-}\right\}=2 \\
& \left\{\gamma_{x}, \gamma_{x}\right\}=\left\{\gamma_{y}, \gamma_{y}\right\}=-2
\end{aligned}
$$

Since the gluon fields are confined to $1+1$ dimensions, there is only one nonvanishing component of the gluon field strength tensor

$$
F_{+-}=-F_{-+}=\partial_{+} A_{-}-\partial_{-} A_{+}+i g_{0}\left[A_{-}, A_{+}\right] .
$$

The QCD part of the theory is simplified if we choose the light cone gauge, where $A_{-}=0$, so that the commutator contained in the field tensor $F_{+-}$disappears and there is only one nonzero component $\left(A_{+}\right)$of the gluon field. The Lagrangian density (2.3) then reduces to

$$
\mathcal{L}_{Q C D}=\frac{1}{2} \operatorname{Tr}\left[\left(\partial_{-} A_{+}\right)^{2}\right]+\sum_{i} \bar{q}_{i}\left(i \partial_{+} \gamma_{-}+i \partial_{-} \gamma_{+}-g_{0} \gamma_{-} A_{+}-m_{0 i}\right) q_{i}
$$

The equation of motion for this field is then

$$
\partial_{-}^{2} A_{+}^{a}=\left(\frac{\partial}{\partial x_{+}}\right)^{2} A_{+}^{a}=-g_{0} \sum_{i} \bar{q}_{i} \lambda^{a} \gamma_{-} q_{i}
$$

The solution of $(\underline{2.16})$ is

$$
A_{+}^{a}\left(x_{+}, x_{-}\right)=g_{0} \int d y_{+} \mathcal{G}\left(x_{+}-y_{+}\right) \sum_{i} \bar{q}_{i}\left(y_{+}, x_{-}\right) \lambda^{a} \gamma_{-} q_{i}\left(y_{+}, x_{-}\right),
$$

where the Green's function $\mathcal{G}$ is

$$
\mathcal{G}\left(x_{+}-y_{+}\right)=-\frac{1}{2}\left|x_{+}-y_{+}\right|+c_{1}\left(x_{+}-y_{+}\right)+c_{2} .
$$

The coefficients $c_{1}$ and $c_{2}$ cannot be determined without knowing the boundary conditions, so they are free parameters. The gauge condition did not eliminate all superfluous degrees of freedom, just as the Coulomb gauge, or the Lorentz gauge, do not determine uniquely the photon propagator in QED (Gribov ambiguity). We can therefore set the coefficients $c_{1}$ and $c_{2}$ equal to zero (a specific choice of gauge) in order to simplify our calculations.

Einhorn discussed the gauge issues related to these two parameters and showed that the eigenvalues of the two body bound state equation are independent of the choice of $c_{1}$ and $c_{2}$. However, the dressed quark mass does depend on the choice of $c_{2}$, and this in turn implies that the location of the quark mass pole is gauge dependent. Since any physically meaningful quantity is gauge invariant, we can conclude that the location of the mass pole is not physically meaningful, and this can happen only if the quarks are confined, so that free quark states do not exist. This is an indirect consequence of confinement. Direct consequences of confinement will be discussed in the next subsection when the two-body bound state equation is discussed. 
Equation (2.17) shows that the gluonic field is no longer a dynamical variable, and that there are therefore no ghosts.

The Fourier transform of the Green's function (2.18) gives us the gluon "propagator", or more precisely the momentum dependence of the effective quark-quark interaction. With $c_{1}$ and $c_{2}$ equal to zero, it can be written

$$
\tilde{\mathcal{G}}\left(k_{-}\right)=\int_{-\infty}^{\infty} d x_{+} e^{i\left(k_{-} x_{+}\right)} \mathcal{G}\left(x_{+}\right) \rightarrow\left\{\frac{1}{k_{-}^{2}}-\delta\left(k_{-}\right) \int_{-\infty}^{\infty} \frac{d \ell_{-}}{\ell_{-}^{2}}\right\} .
$$

This is a singular operator that is well defined only when it is part of an integral over $k_{-}$. The second term in Eq. (2.19) was introduced by Gross and Milana 16] in a different context. Its purpose is to preserve the condition

$$
\mathcal{G}(0)=0=\int_{-\infty}^{\infty} d k_{-} \tilde{\mathcal{G}}\left(k_{-}\right)
$$

and it also insures that the potential is finite for any finite value of $x_{+}$.

The only variables remaining are the quark fields. The Feynman rule for the (undressed) quark propagator is

$$
-i S_{0}(k)=\frac{-i}{m_{0}-k_{-} \gamma_{+}-k_{+} \gamma_{-}-i \epsilon}=-i \frac{m_{0}+k_{-} \gamma_{+}+k_{+} \gamma_{-}}{m_{0}^{2}-2 k_{+} k_{-}-i \epsilon}
$$

and the quark-"gluon" coupling is

$$
-i \mathcal{V}_{q q g}^{a}=-i g_{0} \lambda^{a} \gamma_{-}
$$

leading to the following result for the exchange of gluons with momentum $k_{-}$between quarks with Dirac indicies 1 and 2

$$
\begin{aligned}
-i \mathcal{V}_{q q} & =\sum_{a}\left(-i \mathcal{V}_{q q g}^{a 1}\right) \mathcal{O}_{12}\left(-i \mathcal{V}_{q q g}^{a 2}\right)\left[-i \tilde{\mathcal{G}}\left(k_{-}\right)\right] \\
& =-i g_{0}^{2} \frac{2\left(N_{c}^{2}-1\right)}{N_{c}} \gamma_{-}^{1} \mathcal{O}_{12} \gamma_{-}^{2} \tilde{\mathcal{G}}\left(k_{-}\right) \rightarrow-i g^{2} \gamma_{-}^{1} \mathcal{O}_{12} \gamma_{-}^{2} \tilde{\mathcal{G}}\left(k_{-}\right),
\end{aligned}
$$

where we used Eq. (2.5) (with matrix multiplication of the $\lambda$ matrices implied), and took the limit as $N_{c} \rightarrow \infty$ using the definition (2.6).

The quark self-energy and the dressed quark mass are calculated in the next subsection.

\section{B. Dyson-Schwinger Equation for the dressed quark}

We determine the dressed single quark propagator, $S(p)$, using the (one body) Dyson Schwinger equation (DSE):

$$
\begin{aligned}
S(p) & =S_{0}(p)-S(p) \Sigma(p) S_{0}(p) \\
& =S_{0}(p)-S(p)\left[-i g^{2} \int \frac{d^{2} k}{(2 \pi)^{2}} \tilde{\mathcal{G}}\left(p_{-}-k_{-}\right) \gamma_{-} S(k) \gamma_{-}\right] S_{0}(p)
\end{aligned}
$$

shown graphically in Fig. 1. The gluon interaction does not mix quark flavors, and the

calculation is identical for each flavor of quark, so the flavor index is suppressed. The 


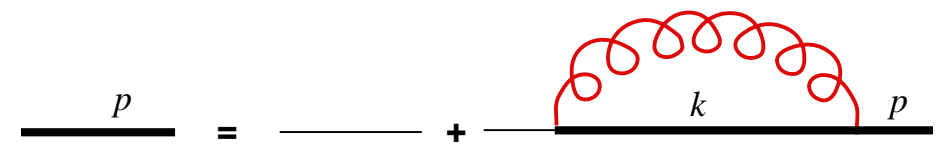

FIG. 1: Dyson-Schwinger equation for the quark. The corkscrew line is the gluon interaction, the thin line the undressed quark propagator, and the heavy solid line the dressed quark propagator.

rainbow approximation (undressed vertices and the absence of the quark loops from the gluon propagator) is justified in the large $N_{c}$ limit [1]. Since for every internal loop there is a factor of $\alpha^{2}=g^{2} /\left(2 N_{c}\right)$, and a multiplicative factor of $\sum_{a} \lambda^{a} \lambda^{a}=2 N_{c}$, the color dependence disappears. The vertex corrections and the quark-gluon vertices do not have a multiplicative factor of $N_{c}$, and are therefore supressed in the large $N_{c}$ limit.

In Eq. (2.24),$d^{2} k=d k_{-} d k_{+}$, and since $D$ does not depend on $k_{+}$, it follows immediately that the self-energy integral does not depend on $p_{+}$either, and must have the form $\Sigma(p)=$ $B\left(p_{-}\right) \gamma_{-}$. Hence the dressed propagator is of the form

$$
S(p)=\frac{1}{m_{0}-p_{-} \gamma_{+}-\left[p_{+}-B\left(p_{-}\right)\right] \gamma_{-}-i \epsilon},
$$

where, using $\gamma_{-} \gamma_{+} \gamma_{-}=2 \gamma_{-}$, the self-energy contribution is then

$$
B\left(p_{-}\right)=-2 i g^{2} \int \frac{d k_{-} d k_{+}}{(2 \pi)^{2}} \frac{k_{-} \tilde{\mathcal{G}}\left(p_{-}-k_{-}\right)}{m_{0}^{2}-2 k_{-}\left(k_{+}-B\left(k_{-}\right)\right)-i \epsilon} .
$$

Performing the $k_{+}$integral gives

$$
\int d k_{+} \frac{k_{-}}{m_{0}^{2}-2 k_{-}\left(k_{+}-B\left(k_{-}\right)\right)-i \epsilon}=\frac{i \pi}{2} \operatorname{sig}\left(k_{-}\right),
$$

and substituting this back into Eq. (2.26) gives

$$
B\left(p_{-}\right)=\frac{g^{2}}{4 \pi} \int d k_{-} \tilde{\mathcal{G}}\left(p_{-}-k_{-}\right) \operatorname{sig}\left(k_{-}\right) .
$$

Using (2.19) for $\tilde{\mathcal{G}}\left(p_{-}-k_{-}\right)$gives

$$
\begin{aligned}
B\left(p_{-}\right) & =\frac{g^{2}}{4 \pi} \int_{-\infty}^{\infty} d k_{-}\left\{\frac{\operatorname{sig}\left(k_{-}\right)}{\left(p_{-}-k_{-}\right)^{2}}-\frac{\operatorname{sig}\left(p_{-}\right)}{\left(p_{-}-k_{-}\right)^{2}}\right\} \\
& =-\frac{g^{2}}{2 \pi p_{-}}
\end{aligned}
$$

Substituting this back into Eq. (2.23) gives

$$
S(p)=\frac{1}{m_{0}-p_{-} \gamma_{+}-\left[p_{+}+\frac{g^{2}}{2 \pi p_{-}}\right] \gamma_{-}-i \epsilon}=\frac{m_{0}+p_{-} \gamma_{+}+\left(p_{+}+\frac{g^{2}}{2 \pi p_{-}}\right) \gamma_{-}}{m_{0}^{2}-\frac{g^{2}}{\pi}-2 p_{+} p_{-}-i \epsilon}
$$


Note that the quark mass pole has been shifted to a smaller value

$$
m_{0}^{2} \rightarrow m^{2}=m_{0}^{2}-\frac{g^{2}}{\pi},
$$

where $m$ is the dressed quark mass. It turns out that choosing $c_{1} \neq 0$ will not effect the dressing of the mass, but choosing $c_{2} \neq 0$ would give

$$
m^{2}=m_{0}^{2}-\frac{g^{2}}{\pi}\left(1-\pi c_{2}\left|p_{-}\right|\right) .
$$

This gives a mass that is momentum dependent and not covariant. Hence the dressed mass is gauge dependent and unphysical. With these cautionary remarks, we choose $c_{1}=c_{2}=0$ because the mass is covariant. One undesirable feature of this choice is that $m^{2}<0$ in the chiral limit. This could be corrected by choosing $c_{2}$ large and positive.

Having obtained the dressed propagator, we are able to proceed with the two body bound state calculation.

\section{Two-body bound states}

\section{Algebraic form of the two body equations}

Consider a bound state of a $q \bar{q}$ pair. The quark has dressed mass $m_{1}$ and electric charge $e_{1}$, and the antiquark (which might be of a different flavor) has dressed mass $m_{2}$ and charge $e_{2}$. The momentum of the bound state is $r$, the momentum of the quark is $p$ and the momentum of the outgoing antiquark is $r-p$, as shown in Fig. 2. In much of the following discussion, we will treat the outgoing antiquark as an incoming quark with momentum $p-r$, and following this convention label the bound state vertex function $\Gamma(p, p-r)$. For a color neutral state we may carry out the color sums using (2.23), giving the Bethe-Salpeter equation for the bound state vertex function:

$$
\Gamma(p, p-r)=i \int \frac{d^{2} k}{(2 \pi)^{2}} V(p, k) \gamma_{-} S_{1}(k) \Gamma(k, k-r) S_{2}(k-r) \gamma_{-},
$$

where $S_{1}$ and $S_{2}$ are dressed quark propagators, and the kernel is the singular operator

$$
V(p, k)=g^{2} \tilde{\mathcal{G}}\left(p_{-}-k_{-}\right) .
$$

With the substitution $\Gamma(p, p-r)=\gamma_{-} G(p, p-r)$ [1], Eq. (2.33) becomes:

$$
G(p, p-r)=4 i \int \frac{d^{2} k}{(2 \pi)^{2}} \frac{V(p, k) k_{-}\left(k_{-}-r_{-}\right) G(k, k-r)}{\left[m_{1}^{2}-k^{2}-i \epsilon\right]\left[m_{2}^{2}-(k-r)^{2}-i \epsilon\right]} .
$$

The formula shows that $G(p, p-r)$ does not depend on $p_{+}$, and hence the $k_{+}$integration can be carried out immediately.

Integrations of the variable $k_{+}$over two quark propagators occurr frequently, and are worked out in detail in Appendix $\mathrm{A}$. It is convenient to introduce the momentum fractions

$$
y=\frac{k_{-}}{r_{-}} \quad z=\frac{p_{-}}{r_{-}} .
$$




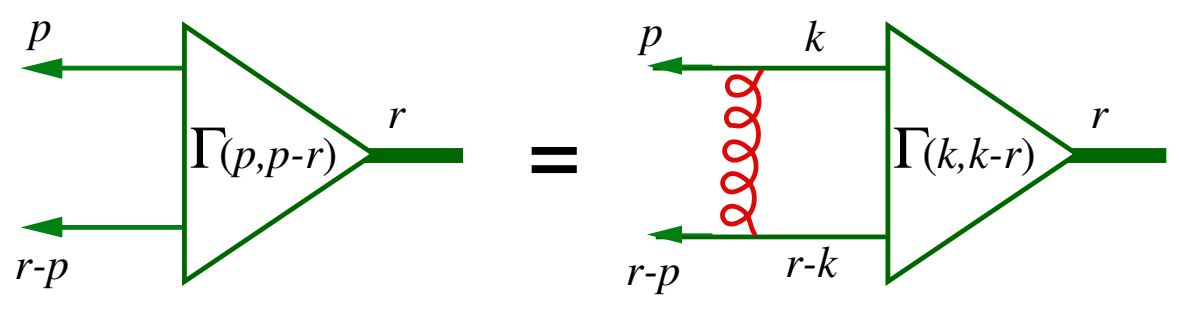

FIG. 2: The Bethe-Salpeter equation

If the fraction $z$ lies in the interval [0,1], Eq. (2.35) reduces to

$$
\begin{aligned}
\mu_{n}^{2} \Phi_{n}(z, r) & =\left(\frac{\alpha_{1}+1}{z}+\frac{\alpha_{2}+1}{1-z}\right) \Phi_{n}(z, r)-f_{0}^{1} d y \frac{\Phi_{n}(y, r)-\Phi_{n}(z, r)}{(z-y)^{2}} \\
& \equiv \frac{\pi}{g^{2}} H(z) \Phi_{n}(z, r) \quad(\text { if } z \in[0,1]),
\end{aligned}
$$

where $f$ is the principal value integral, and $\mu_{n}, \alpha_{1}$, and $\alpha_{2}$ are dimensionless parameters

$$
\mu_{n}^{2}=\frac{\pi M_{n}^{2}}{g^{2}}, \quad \alpha_{1}=\frac{\pi m_{1}^{2}}{g^{2}}, \quad \alpha_{2}=\frac{\pi m_{2}^{2}}{g^{2}},
$$

with $r^{2}=M_{n}^{2}$ the anticipated mass eigenvalues that will emerge from the solution of the equation, and $m_{i}$ the dressed quark masses. The two-body "wave function" $\Phi_{n}(z, r)$, is defined by

$$
\Phi_{n}(z, r)=\frac{\mathcal{N} r_{-} G_{n}(z, r)}{\Delta\left(z, M_{n}^{2}\right)}=\frac{\mathcal{N} r_{-} G_{n}(z, r)}{\left(\frac{m_{1}^{2}}{z}+\frac{m_{2}^{2}}{1-z}-M_{n}^{2}\right)}=\frac{\mathcal{N} r_{-} G_{n}(z, r)}{\frac{g^{2}}{\pi}\left(\frac{\alpha_{1}}{z}+\frac{\alpha_{2}}{1-z}-\mu_{n}^{2}\right)}
$$

where $G_{n}(z, r)=G(p, p-r)$ is the anticipated eigenfunction solution for the vertex corresponding to the $n$th bound state, and $\mathcal{N}$ is a normalization constant. It is chosen so that

$$
1=\int_{0}^{1} d z \Phi_{n}^{2}(z, r),
$$

and will be calculated in subsection IIIC, Note that Eq. (2.37) defines the two-quark Hamiltonian, $H(z)$, on the interval $0 \leq z \leq 1$. The bare quark masses enter into the Hamiltonian, while the dressed quark masses enter into the relation (2.39) between the vertex function and the wave function.

If the momentum fraction $z$ lies outside of the interval $0 \leq z \leq 1$, the vertex function defined by Eq. (2.35) is not zero, but can be obtained by quadrature from the vertex function defined inside of the interval $[0,1]$. Using (A8) and the definition (2.39) gives

$$
\Phi_{n}(z, r)=\frac{1}{\left(\frac{\alpha_{1}}{z}+\frac{\alpha_{2}}{1-z}-\mu_{n}^{2}\right)} \int_{0}^{1} d y \frac{\Phi_{n}(y, r)}{(z-y)^{2}} \quad(\text { if } z \notin[0,1]) .
$$


These definitions and equations will be used in the following sections when the hadronic currents are computed.

An alternative form of Eq. (2.37) follows if we integrate the last term by parts:

$$
\begin{aligned}
\mu_{n}^{2} \Phi_{n}(z, r)= & \left(\frac{\alpha_{1}+1}{z}+\frac{\alpha_{2}+1}{1-z}\right) \Phi_{n}(z, r)+f_{0}^{1} d y \frac{\Phi_{n}^{\prime}(y, r)}{(z-y)} \\
& +\left(\frac{\Phi_{n}(0, r)-\Phi_{n}(z, r)}{z}\right)+\left(\frac{\Phi_{n}(1, r)-\Phi_{n}(z, r)}{1-z}\right),
\end{aligned}
$$

where $\Phi_{n}^{\prime}(y, r)=\partial \Phi(y, r) / \partial y$. If $\Phi_{n}(0, r)=\Phi_{n}(1, r)=0$, which is true in all but the chiral limit, this form of the equation follows directly from 't Hooft's principle value prescription [1], so that our two-body equation is identical with 't Hooft's, even though we have finite dressed quark masses.

To avoid confusion later, we call attention to the fact that the wave function defined by Eqs. (2.39) and (2.41) is not identical to the Bethe-Salpeter wave function. The latter can be defined

$$
\Psi(p, r) \equiv \frac{\mathcal{N} p_{-}(p-r)_{-} G(p, p-r)}{\left(m_{1}^{2}-p^{2}-i \epsilon\right)\left(m_{2}^{2}-(p-r)^{2}-i \epsilon\right)},
$$

where the factor $p_{-}(p-r)_{-}$in the numerator comes from the $\gamma_{-}$factors in the quark propagators $S$. This wave function carries the $p_{+}$dependence of the quark propagators. If we integrate (2.43) over $p_{+}$(using the fact that $G$ does not depend on $p_{+}$) we obtain

$$
\psi\left(p_{-}, r\right) \equiv \int_{-\infty}^{\infty} d p_{+} \Psi(p, r)=\left\{\begin{array}{ll}
\frac{\mathcal{N} r_{-} G(p, p-r)}{\Delta\left(z, r^{2}\right)}=\Phi(z, r) & \text { if } z \in[0,1] \\
0 & \text { if } z \notin[0,1]
\end{array} .\right.
$$

This is the "equal $t_{-}$" wave function, and equals the wave function we are using only in the region $z \in[0,1]$. As we have seen, the wave function $\Phi(z, r)$ that solves the 't Hooft equation is non zero for all $z$.

We conclude this discussion of the two body wave equation by returning to Eq. (2.33). In place of the replacement $\Gamma(p, p-r)=\gamma_{-} G_{n}(z, r)$, it could equally well have been assumed that

$$
\Gamma(p, p-r)=\gamma_{-} \gamma_{k} G_{n}(z, r),
$$

where $k$ is either $x$ or $y$ (recall that we are assuming that the Dirac matrices live in $1+3$ dimensional space). Note that the Bethe-Salpeter equations with the substitutions (2.45) reduce to the same equation (2.35) for $G_{n}$, and hence each two-body state is triplely degererate, with spin structure given by $\gamma_{-}, \gamma_{-} \gamma_{x}$, or $\gamma_{-} \gamma_{y}$. In each of these three cases the wave functions and bound state mass are identical. The additional states with the $\gamma_{x}$ or $\gamma_{y}$ structure are outside the scope of the original 't Hooft model, but must be considered in reduced QCD. They are states with quark spins in the transverse direction, and will play a major role in the discussion of DIS below.

The chiral limit is exactly soluable and of some interest. If $m_{0 i}=0$, then Eq. (2.37) has the normalized ground state solution

$$
M_{0}=0, \quad \Phi_{0}(z, r)=1 .
$$

Outside of the interval $[0,1]$, Eq. (2.41) gives the same result, so that

$$
\Phi_{0}(z, r)=1
$$

for all $z$ in $(-\infty, \infty)$. 


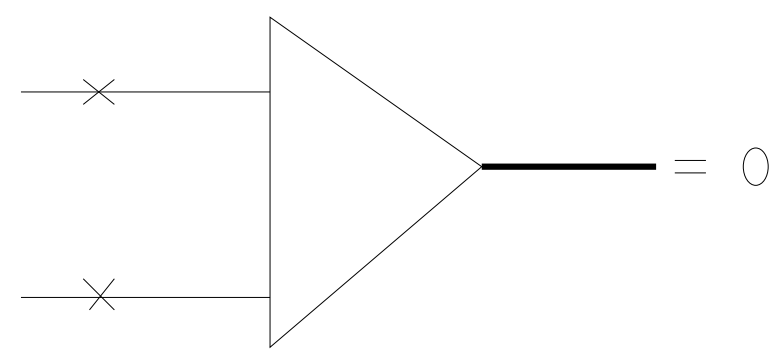

FIG. 3: Diagrammatic representation of confinement via two-body interaction. The $\times$ indicates that the dressed quark is on-shell, and at the point when both quarks are on-shell, the vertex function is zero.

\section{Confinement in the 't Hooft model}

The 't Hooft model illustrates how confinement can be realized in two (apparently different) ways. If the singularities of the confining interaction are left unregularized, as they were in 't Hooft's original paper [1], the quark masses are infinite. Even if the singularity is regularized as in this paper, whenever $m_{0 i}^{2}<g^{2} / \pi$ the dressed quark mass will be unphysical, and there is no real quark mass pole. This insures that the quarks are confined.

In the second case $\left(m_{0 i}^{2}>g^{2} / \pi\right.$ and $\left.m_{i}^{2}>0\right)$ the dressed quark mass is real and positive and the there is a quark mass pole. What keeps the quarks confined in this case? The answer is that the quark vertex function $G_{n}(z, r)$ has a zero at precisely the value of $z$ where all of the quarks in the bound state could be on mass shell. This requirement is illustrated diagramatically in Fig. 3, and seems miraculous. However, as discussed in Ref. [17], it is only a consequence of fact that the wave function is finite at the pole (because the wave equation permits no singularities), and therefore the vertex function

$$
G_{n}(z, r)=\left[\frac{m_{1}^{2}}{z}+\frac{m_{2}^{2}}{1-z}-M_{n}^{2}\right] \frac{\Phi(z, r)}{\mathcal{N} r_{-}}
$$

must be zero. Precisely the same mechanism works in the Schrödinger description of two nonrelativistic particles bound by a linear (or any other) confining potential.

In summary, quark mass poles are always unphysical because the dressed mass is gauge dependent, and bound state poles are physical because they are gauge invariant. In this respect the undressed quark mass is the only physically significant parameter. The two ways of viewing confinement are therefore not exclusive, but equivalent, since they are related through gauge transformations.

\section{Numerical solutions for meson wave functions}

The bound state equation was solved numerically using modified cubic splines. The splines and the technique are fully described in Appendix G. Each spline is a smooth function (continuous up to its third derivative) and has support over a small region of the $z$ axis. The splines overlap, so that by independently adjusting the height of each spline it is possible to smoothly approximate any function defined on the interval $[0,1]$.

We have modified the standard spline technique to insure that the numerical solutions satisfy the boundary conditions exactly. These boundary conditions are determined by examing Eq. (2.37) in the vicinity of $z \sim 0$ and $z \sim 1$ [1, 11], and require the wave function 
TABLE I: Numerical values of $M_{n}^{2}$ for $m_{01}=m_{02}=1.5$ and various $n_{s}$ (number of splines) and $n_{g}$ (number of guass points for each spline segment).

\begin{tabular}{|c|c|c|c|c|c|c|c|c|c|c|}
\hline level & $n_{s}$ & & 20 & & & 40 & & & 60 & \\
\hline$n$ & $n_{g}$ & 2 & 4 & 6 & 2 & 4 & 6 & 2 & 4 & 6 \\
\hline 1 & & 13.730 & 13.732 & 13.732 & 13.717 & 13.718 & 13.718 & 13.714 & 13.715 & 13.715 \\
\hline 2 & & 25.613 & 25.614 & 25.615 & 25.572 & 25.573 & 25.573 & 25.563 & 25.564 & 25.564 \\
\hline 10 & & 108.95 & 109.35 & 109.36 & 108.42 & 108.43 & 108.43 & 108.28 & 108.28 & 108.28 \\
\hline 20 & & 186.17 & 210.60 & 210.58 & 208.82 & 209.69 & 209.69 & 208.88 & 208.97 & 208.97 \\
\hline 30 & & - & - & - & 299.64 & 314.02 & 314.02 & 308.04 & 309.38 & 309.38 \\
\hline 40 & & - & - & - & 362.04 & 409.77 & 409.74 & 410.31 & 411.24 & 411.24 \\
\hline
\end{tabular}

TABLE II: Numerical values of $M_{n}^{2}$ for $m_{01}=m_{02}=0.5$ and various $n_{s}$ and $n_{g}$.

\begin{tabular}{rcccccccccc} 
level & $n_{s}$ & \multicolumn{3}{c}{20} & \multicolumn{9}{c}{40} & \multicolumn{3}{c}{60} \\
& $n_{g}$ & 4 & 6 & 8 & 4 & 6 & 8 & 4 & 6 & 8 \\
\hline 1 & & 2.6196 & 2.6625 & 2.6841 & 2.6485 & 2.6786 & 2.6936 & 2.6616 & 2.6858 & 2.6979 \\
2 & & 10.540 & 10.650 & 10.706 & 10.616 & 10.692 & 10.731 & 10.648 & 10.710 & 10.741 \\
10 & & 86.170 & 86.371 & 86.477 & 86.055 & 86.288 & 86.409 & 86.197 & 86.400 & 86.503 \\
20 & & 199.44 & 199.43 & 199.43 & 183.93 & 184.11 & 184.21 & 183.53 & 183.77 & 183.90 \\
30 & & - & - & - & 287.16 & 287.22 & 287.25 & 282.09 & 282.27 & 282.36 \\
40 & & - & - & - & 399.18 & 399.15 & 399.15 & 383.13 & 383.22 & 383.27
\end{tabular}

go like

$$
\begin{aligned}
& \Phi_{n}(z, r) \rightarrow z^{\beta_{1}} \quad(\text { as } z \rightarrow 0) \quad \text { with } \pi \beta_{1}=-\alpha_{1} \tan \left(\pi \beta_{1}\right) \\
& \Phi_{n}(z, r) \rightarrow(1-z)^{\beta_{2}} \quad(\text { as } z \rightarrow 1) \text { with } \pi \beta_{2}=-\alpha_{2} \tan \left(\pi \beta_{2}\right) .
\end{aligned}
$$

Note that the $\beta_{i}=0$ in the chiral limit (when $\alpha_{i}=-1$ ), consistent with the exact solution (2.47). To fit these boundary conditions the splines closest to $z=0$ and $z=1$ have these fractional powers built into their functional form, as described in Appendix G.

The wave function is expanded into $n_{s}$ splines, each with four segments (except for those at the boundary, which have only three). The splines overlap, and taking into account that there must be at least three splines (one regular spline in the center and one modifed spline at each end), the total number of segments is $n_{s}+1$, dividing the interval into segments of length $1 /\left(n_{s}+1\right)$. The equation is turned into a matrix equation by integrating over each segment using $n_{g}$ gaussian points. Tables $\llbracket$ and $\amalg$ show the numerical stability. If the quark mass is larger than unity, better than $1 \%$ accuracy is achieved with only two gauss points per interval, provided $n \lesssim n_{s}$. As the level number $n$ increases above $\sim n_{s} / 2$ toward $n_{s}$, the reliability of the calculation decreases. To study highly excited states, it is sufficient to use $n_{g}=2$, and push $n_{s}$ as high as possible.

The situation is somewhat different if the bare quark masses are less than unity (Table III). In this case the wave function is very steep at the boundaries and 4 or 6 gaussian points per interval are needed to get $1 \%$ accuracy for the low lying states. In a subsequent paper [15], we will limit our numerical discussion of duality to cases with $m_{0 i}>1$ where the solutions are very stable, and there are no poles in the region outside of $[0,1]$ [see the brief remarks following Eq. ([F14)]. 


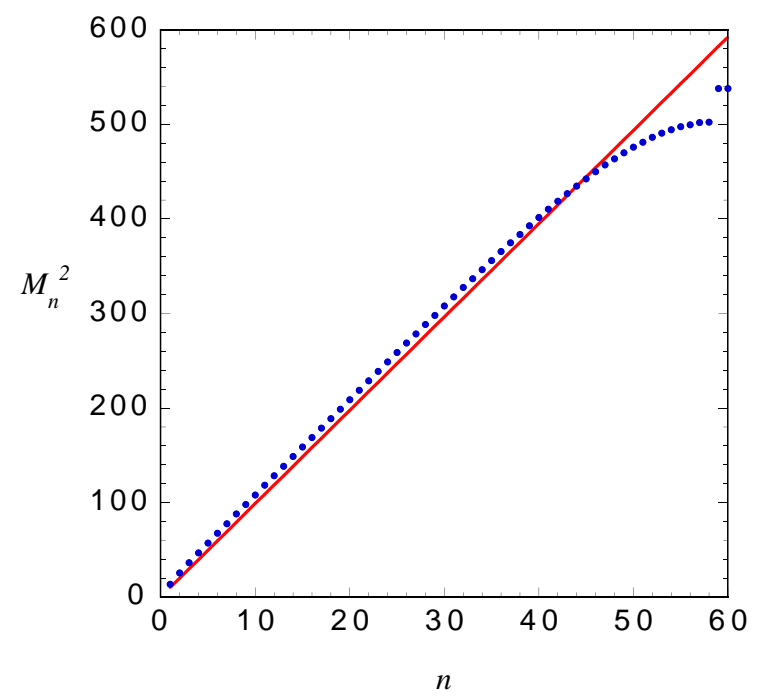

FIG. 4: The square of the bound state mass (in units of $g^{2} / \pi$ ) versus the order of the state $n$, computed using 60 splines. The straight line is the linear relation $n \pi g^{2}$. Note that the masses follow this linear relation up to near $n \sim n_{s}=60$, where the departure from linearity is due to numerical inaccuracy.

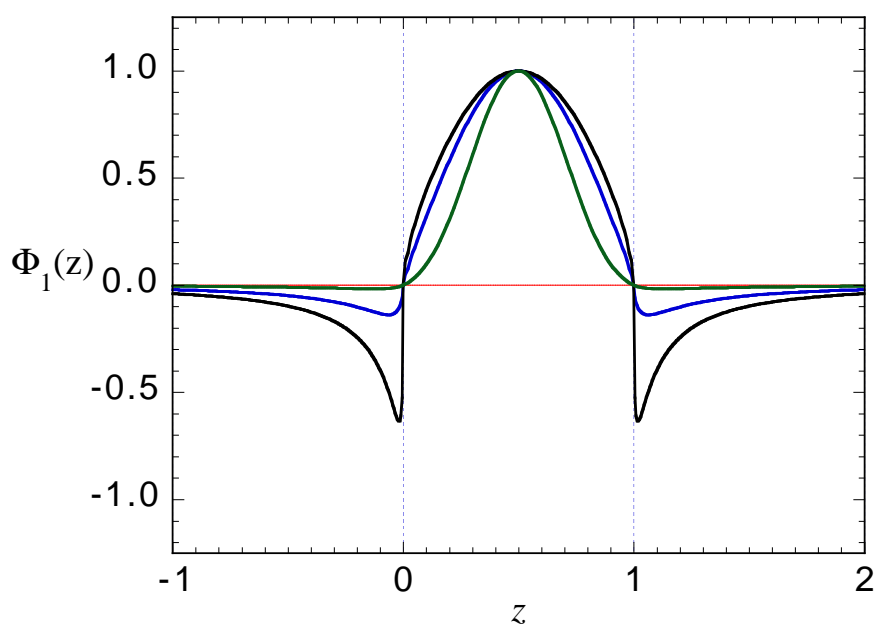

FIG. 5: The ground state wave functions for equal bare quark masses $m_{0}$ of $1.1,1.5$, and 3 (the wave functions become steeper at $z=0$ and $z=1$ as the quark mass decreases). In all cases 60 splines were used, and the wave function was normalized to a maximum value of unity.

Figure 4 shows the linearly rising Regge trajectory characteristic of the 't Hooft model. Figures 5 [17 show the ground, first excited and 10th level for three cases with equal quark masses of different values. Note the "tails" of the wave functions outside of the region $[0,1]$, given by Eq. (2.41). These tails are large for small quark masses, but shrink quickly as the quark mass increases, and are also small for highly excited states. Figure 8 shows the 20 th level for spline numbers 20,40 , and 60 . Note that the state is not fully described even with 40 splines, supporting the observation that an accurate description of states of order $n$ requires a spline number of approximately $\sim 2 n$. 


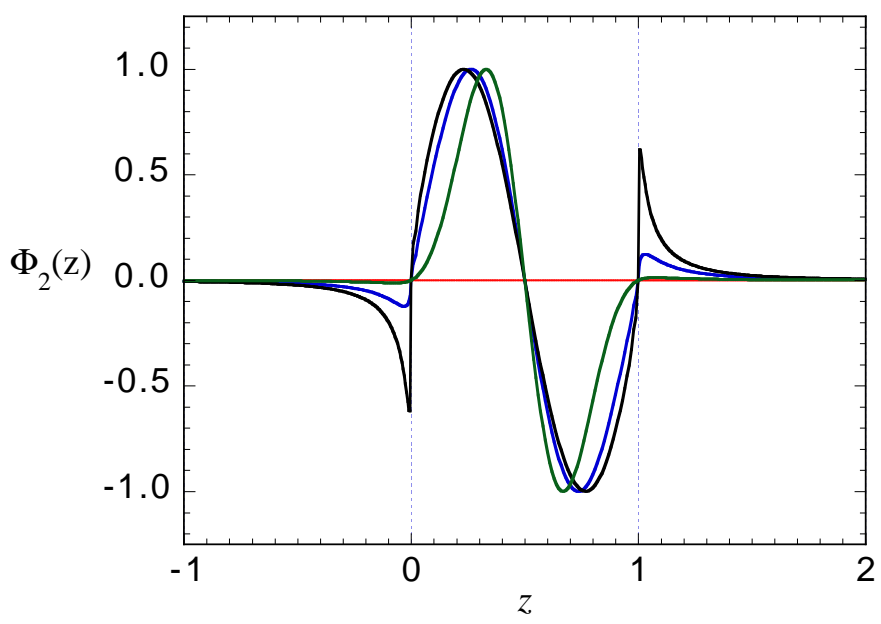

FIG. 6: The second state (first excited state) for the same three quark masses shown in Fig. [5 (See caption to Fig. 囵)

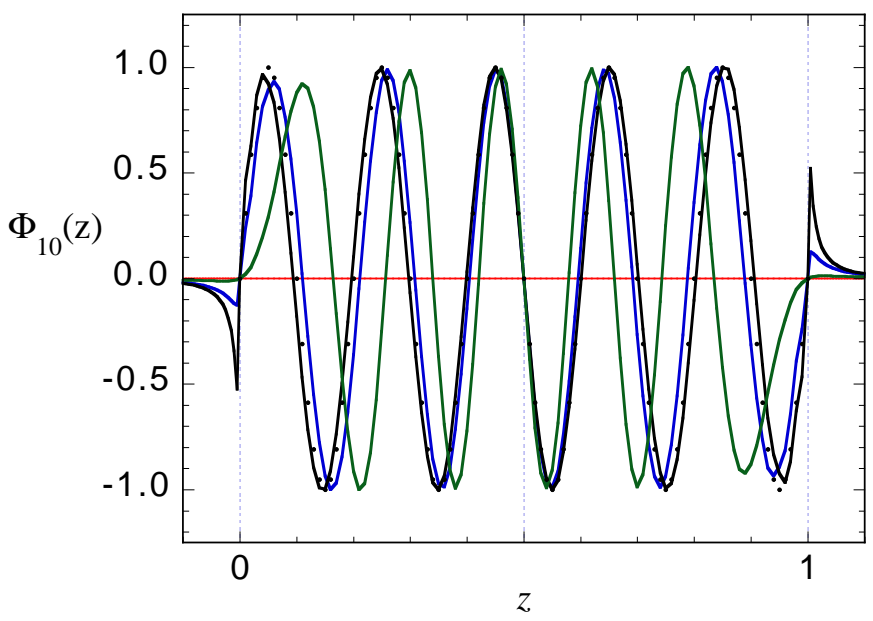

FIG. 7: The tenth state for the same three quark masses shown in Fig. 5 (See caption to Fig. 5 ) In this figure the dots are $\sin (10 \pi z)$, showing that this is an excellent approximation for small quark masses.

\section{Scattering of quarks and antiquarks in the presence of confinement}

\section{Completeness relation and the two-body Greens function}

The Hamiltonian defined in Eq. (2.37) is hermitian on the interval $[0,1]$ and it is straightforward to show that the different solutions are orthogonal on this interval. With the normalization given in Eq. (2.40)

$$
\int_{0}^{1} d z \Phi_{n}\left(z, r_{n}\right) \Phi_{m}\left(z, r_{m}\right)=\delta_{n m} .
$$




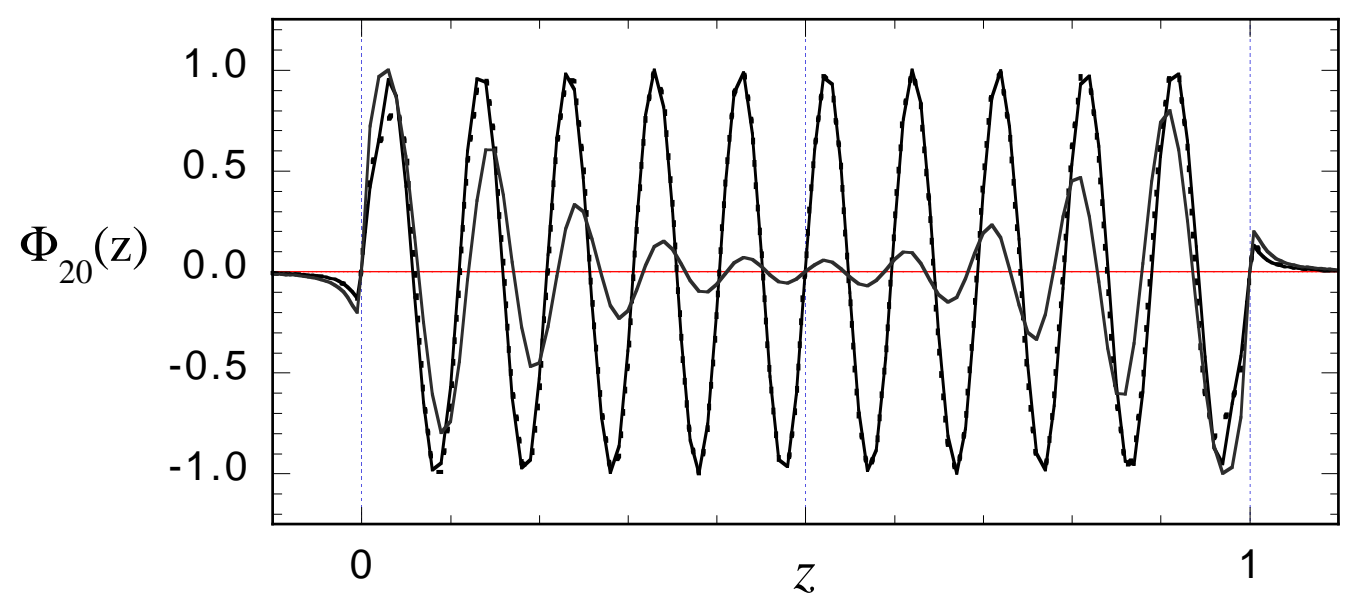

FIG. 8: The 20th state for and equal quark mass of 1.5. The small amplitude curve was calculated for $n_{s}=20$, and completely fails to represent the state. The dotted and full amplitude solid lines are calculated for $n_{s}=40$ and 60 , respectively. Note that $n_{s}=40$ does well except for the first and last oscillation.

The completeness of the eigenfunctions implies that

$$
\sum_{n} \Phi_{n}\left(z, r_{n}\right) \Phi_{n}\left(z^{\prime}, r_{n}\right)=\delta\left(z-z^{\prime}\right) \quad \text { if } z, z^{\prime} \in[0,1]
$$

The Greens function is therefore

$$
G\left(z, z^{\prime}, q^{2}\right)=\sum_{n} \frac{\Phi_{n}\left(z, r_{n}\right) \Phi_{n}\left(z^{\prime}, r_{n}\right)}{q^{2}-M_{n}^{2}},
$$

where $q$ is any external momentum. The completness relation shows that $G$ has the property of a Greens function

$$
\begin{aligned}
\left\{q^{2}-H(z)\right\} G\left(z, z^{\prime}, q^{2}\right) & =\sum_{n}\left(q^{2}-H(z)\right) \frac{\Phi_{n}\left(z, r_{n}\right) \Phi_{n}\left(z^{\prime}, r_{n}\right)}{q^{2}-M_{n}^{2}} \\
& =\delta\left(z-z^{\prime}\right) \quad \text { if } z, z^{\prime} \in[0,1] .
\end{aligned}
$$

The same argument also gives

$$
G\left(z, z^{\prime}, q^{2}\right)\left\{q^{2}-H\left(z^{\prime}\right)\right\}=\delta\left(z-z^{\prime}\right) \quad \text { if } z, z^{\prime} \in[0,1] .
$$

\section{The scattering matrix}

In preparation for the study of electromagnetic interactions, it is useful to introduce the $q \bar{q}$ scattering matrix, defined by the following infinite series

$$
\begin{aligned}
& M\left(p^{\prime}, p ; q\right) \gamma_{1-} \otimes \gamma_{2-}=V\left(p^{\prime}, p\right) \gamma_{1-} \otimes \gamma_{2-} \\
& \quad+i \int \frac{d^{2} k}{(2 \pi)^{2}} V\left(p^{\prime}, k\right) V(k, p)\left[\gamma_{-} S_{1}(k) \gamma_{-}\right]_{1} \otimes\left[\gamma_{-} S_{2}(k-q) \gamma_{-}\right]_{2}
\end{aligned}
$$




$$
\begin{aligned}
& +i^{2} \int \frac{d^{2} k^{\prime}}{(2 \pi)^{2}} \int \frac{d^{2} k}{(2 \pi)^{2}} V\left(p^{\prime}, k^{\prime}\right) V\left(k^{\prime}, k\right) V(k, p) \\
& \quad \times\left[\gamma_{-} S_{1}\left(k^{\prime}\right) \gamma_{-} S_{1}(k) \gamma_{-}\right]_{1} \otimes\left[\gamma_{-} S_{2}(k-q) \gamma_{-} S_{2}\left(k^{\prime}-q\right) \gamma_{-}\right]_{2} \\
& +\cdots
\end{aligned}
$$

The momentum are defined as for the two-body bound state, except that here $q$ is the unconstrained momentum of the $q \bar{q}$ pair and $q^{2}$ is not an eigenvalue. The kernel $V$ is the singular operator (2.34). The singularities of this operator will never be exposed because we will limit application of this scattering equation to cases where we integrate over either the initial or final momentum. Note that the structure of the equation anticipates the reduction of the dressed propagators

$$
\left[\gamma_{-} S_{i}(k) \gamma_{-}\right]_{i}=\frac{k_{-}\left[\gamma_{-} \gamma_{+} \gamma_{-}\right]_{i}}{m_{i}^{2}-2 k_{-} k_{+}}=\frac{2 k_{-}}{m_{i}^{2}-2 k_{-} k_{+}} \gamma_{i-}
$$

so that the factor of $\gamma_{1-} \otimes \gamma_{2-}$ is common, and the series (2.55) reduces to

$$
\begin{aligned}
M\left(p^{\prime}, p ; q\right)=V\left(p^{\prime}, p\right) & +i \int \frac{d^{2} k}{\pi^{2}} \frac{V\left(p^{\prime}, k\right) V(k, p)}{d_{2}\left(k_{-}, k_{+}, q\right)} \\
& +i^{2} \int \frac{d^{2} k^{\prime}}{\pi^{2}} \int \frac{d^{2} k}{\pi^{2}} \frac{V\left(p^{\prime}, k^{\prime}\right) V\left(k^{\prime}, k\right) V(k, p)}{d_{2}\left(k_{-}^{\prime}, k_{+}^{\prime}, q\right) d_{2}\left(k_{-}, k_{+}, q\right)}+\cdots,
\end{aligned}
$$

where $d_{2}$ was defined in Eq. (A33). From this result we can conclude that $M$ does not depend on $p_{+}^{\prime}$ and $p_{+}$.

In applications below, we will encounter this scattering series in the following form

$$
\begin{aligned}
\langle M G \mathcal{O}\rangle\left(p^{\prime} ; q\right)= & \int \frac{d^{2} p}{\pi^{2}} \frac{M\left(p^{\prime}, p ; q\right) \mathcal{O}\left(p_{-}, q\right)}{d_{2}\left(p_{-}, p_{+}, q\right)}=\int \frac{d^{2} p}{\pi^{2}} \frac{V\left(p^{\prime}, p\right) \mathcal{O}\left(p_{-}, q\right)}{d_{2}\left(p_{-}, p_{+}, q\right)} \\
& +i \int \frac{d^{2} k}{\pi^{2}} \frac{V\left(p^{\prime}, k\right)}{d_{2}\left(k_{-}, k_{+}, q\right)} \int \frac{d^{2} p}{\pi^{2}} \frac{V(k, p) \mathcal{O}\left(p_{-}, q\right)}{d_{2}\left(p_{-}, p_{+}, q\right)} \\
& +i^{2} \int \frac{d^{2} k^{\prime}}{\pi^{2}} \int \frac{d^{2} k}{\pi^{2}} \frac{V\left(p^{\prime}, k^{\prime}\right) V\left(k^{\prime}, k\right)}{d_{2}\left(k_{-}^{\prime}, k_{+}^{\prime}, q\right) d_{2}\left(k_{-}, k_{+}, q\right)} \int \frac{d^{2} p}{\pi^{2}} \frac{V(k, p) \mathcal{O}\left(p_{-}, q\right)}{d_{2}\left(p_{-}, p_{+}, q\right)}
\end{aligned}
$$

where $\mathcal{O}\left(p_{-}, q\right)$ is an operator that, by assumption, does not depend on $p_{+}$. In Appendix C we show how to use the Greens function to write this series as

$$
\int_{0}^{1} d z \mathrm{M}\left(z^{\prime}, z ; q^{2}\right) \frac{\mathrm{O}\left(z, q^{2}\right)}{\Delta\left(z, q^{2}\right)}=\sum_{n} \Delta\left(z^{\prime}, M_{n}^{2}\right) \frac{\Phi_{n}\left(z^{\prime}, r_{n}\right)}{M_{n}^{2}-q^{2}} \int_{0}^{1} d z \Phi_{n}\left(z, r_{n}\right) \mathrm{O}\left(z, q^{2}\right),
$$

where we replaced $\left(q_{-}\right)^{2} M\left(p^{\prime}, p ; q\right) / \pi \rightarrow \mathrm{M}\left(z^{\prime}, z ; q^{2}\right)$ and $\mathcal{O}\left(p_{-}, q\right) \rightarrow \mathrm{O}\left(z, q^{2}\right)$.

This equation has a nice physical interpretation. It displays the "scattering amplitude" as the sum over the propagation of the bound states, which is all confinement will allow (without meson decay mechanisms, which are ignored in this paper, no cuts are possible). The only singularities in $q^{2}$ that can occur are poles at the bound state masses $M_{n}^{2}$.

With these tools in place we are able to study the electromagnetic interaction of hadrons. 


\section{REDUCED QCD AND THE ELECTROMAGNETIC SECTOR}

We return to the discussion of the Lagrangian density introduced in Eq. (2.2) and look at the electromagnetic sector. In rQCD the electromagnetic fields (and the electron currents) are extended to $1+3$ dimensions. The Lagrangian density is therefore

$$
\begin{aligned}
\mathcal{L}_{Q E D}(t, \mathbf{r})=-\frac{1}{4} F_{0}^{\mu \nu}(t, \mathbf{r}) F_{0 \mu \nu}(t, \mathbf{r}) & +\sum_{i} e_{i} \bar{q}_{i}(t, z) \gamma_{\mu} q_{i}(t, z) A_{0}^{\mu}(t, \mathbf{r}) \\
& -e J_{\mu}^{e}(t, \mathbf{r}) A_{0}^{\mu}(t, \mathbf{r}),
\end{aligned}
$$

where $A_{0}(t, \mathbf{r})$ is the electromagnetic field (with the subscript 0 distinguishing it from the gluon field), the vector sums are now over 4 dimensions with $\mu=\{0,1,2,3\}, e_{i}$ is the electromagnetic charge of quarks with flavor $i, J_{\mu}^{e}(t, \mathbf{r})$ is the electron current that produces the electromagnetic field, and

$$
F_{0}^{\mu \nu}=\partial^{\mu} \mathcal{A}_{0}^{\nu}-\partial^{\nu} \mathcal{A}_{0}^{\mu},
$$

is the electromagnetic field tensor. Because the electron current exists in four dimensions, all four components of the electromagnetic field will be non-zero, in general, and all four components of the quark current will be excited by electromagnetic scattering.

We now apply this discussion to electron scattering, where $Q^{2}=-q^{2}>0$, so that $q_{-}<0$. The initial particle (quark or hadron) has momentum $p=p^{\prime}-q$ and the final particle momentum $p^{\prime}$, with momentum fractions

$$
z^{\prime}=\frac{p_{-}^{\prime}}{q_{-}} \quad \frac{p_{-}}{q_{-}}=z^{\prime}-1
$$

If the final particle is on shell, then $p_{-}^{\prime}>0$ and $z^{\prime}<0$, and $z^{\prime} \rightarrow-\infty$ as $Q^{2} \rightarrow 0$. We will use the notation $j_{0}^{\mu}$ to denote the bare quark current operator, which is

$$
j_{0}^{\mu}=\gamma^{\mu} .
$$

While the dressing of the strong quark-gluon vertex is supressed by the large $N_{c}$ limit [1], the quark electromagnetic vertex can be dressed by gluon exchanges. The longitudinal part of the dressed electromagnetic vertex $\left(j^{\mu}\right)$ can be computed directly from the WardTakahashi identity

$$
q_{\mu} j^{\mu}\left(p^{\prime}, p\right)=S^{-1}(p)-S^{-1}\left(p^{\prime}\right)
$$

where $p+q=p^{\prime}$. Using the dressed propagator (2.30), we find that

$$
\begin{aligned}
& j_{-}=\gamma_{-} \\
& j_{+}=\gamma_{+}-\gamma_{-} \frac{g^{2}}{2 \pi} \frac{1}{p_{-}^{\prime} p_{-}}=\gamma_{+}+\gamma_{-} \frac{g^{2}}{2 \pi} \frac{1}{q_{-}^{2} z^{\prime}\left(1-z^{\prime}\right)} .
\end{aligned}
$$

Consequently the $j_{-}$component is unmodified.

\section{A. Electromagnetic coupling to quarks and the quark form factor}

It is instructive to obtain the full quark current directly from the dressing of the quarkphoton vertex. The vertex is dressed by successive gluon exchanges, as shown diagramatically in Fig. 9. When coupling to a photon, there is no flavor change, and hence, for each 


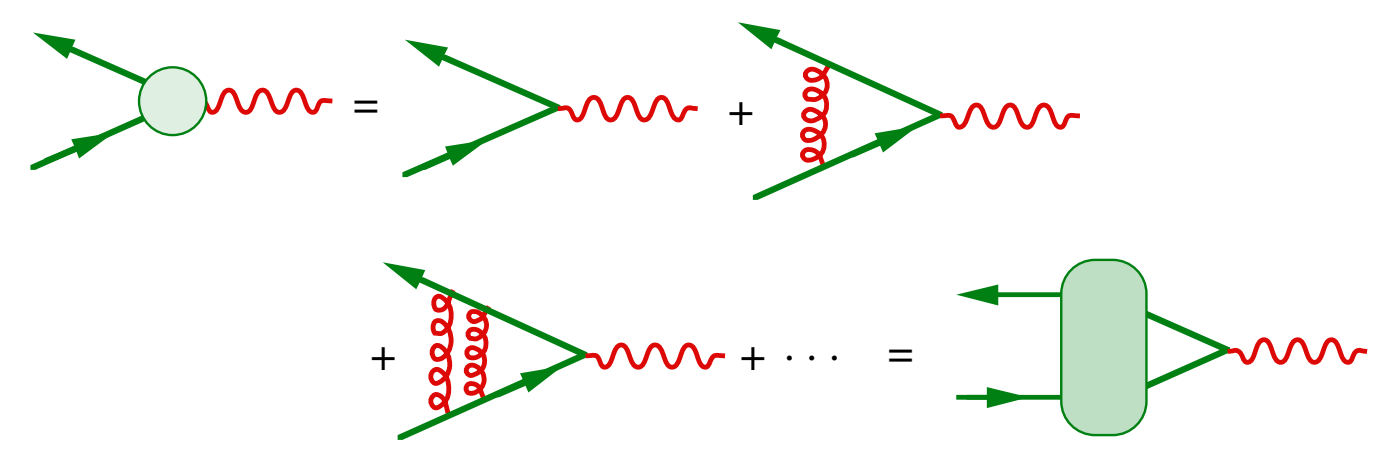

FIG. 9: Diagrammatic picture of the gluonic dressing of the quark current.

flavor $i$, this series is

$$
\begin{aligned}
& j_{i}^{\mu}\left(p^{\prime}, p^{\prime}-q\right)=\gamma^{\mu}+i \int \frac{d^{2} k}{(2 \pi)^{2}} V\left(p^{\prime}, k\right) \gamma_{-} S_{i}(k) \gamma^{\mu} S_{i}(k-q) \gamma_{-} \\
& \quad+i^{2} \int \frac{d^{2} p}{(2 \pi)^{2}} \int \frac{d^{2} k}{(2 \pi)^{2}} V\left(p^{\prime}, p\right) V(p, k) \gamma_{-} S_{i}(p) \gamma_{-} S_{i}(k) \gamma^{\mu} S_{i}(k-q) \gamma_{-} S_{i}(p-q) \gamma_{-} \\
& \quad+\cdots
\end{aligned}
$$

This series is evaluated in Appendix D using the methods developed in Appendix C. The final results are conveniently expressed in term of a quark form factor, defined to be

$$
F_{i}\left(z^{\prime}, Q^{2}\right) \equiv \sum_{n} \Delta_{i}\left(z^{\prime}, M_{n}^{2}\right) \frac{\Phi_{n}\left(z^{\prime}, r_{n}\right)}{M_{n}^{2}+Q^{2}} \int_{0}^{1} d y \Phi_{n}\left(y, r_{n}\right)
$$

where $z^{\prime}=p_{-}^{\prime} / q_{-}$and $Q^{2}=-q^{2}$ in anticipatation of applications to electron scattering. Note that the form factor is expressed as a sum of bound state poles at $Q^{2}=-M_{n}^{2}$, arising from the propagation of the bound states that couple to the photon. Vector dominance is a rigorous consequence of this model.

Using this form factor, the currents reduce to

$$
\begin{aligned}
& j_{i-}\left(p^{\prime}, q\right)=\gamma_{-}+\gamma_{-} F_{i}\left(z^{\prime}, Q^{2}\right) \\
& j_{i+}\left(p^{\prime}, q\right)=\gamma_{+}-\gamma_{-}\left\{\frac{g^{2}}{2 \pi} \frac{1}{p_{-}^{\prime} p_{-}}+\frac{q_{+}}{q_{-}} F_{i}\left(z^{\prime}, Q^{2}\right)\right\} \\
& j_{i}^{x}\left(z^{\prime}, q^{2}\right)=\gamma_{x}-\gamma_{x} \gamma_{-}\left\{\frac{q_{-}}{m_{0 i}} \frac{g^{2}}{2 \pi} \frac{1}{p_{-}^{\prime} p_{-}}+\frac{q_{+}}{m_{0 i}} F_{i}\left(z^{\prime}, Q^{2}\right)\right\} .
\end{aligned}
$$

Note that the terms independent of the quark form factor $F_{i}$ are identical to the expected result (3.6), and that the contribution from the form factor is purely transverse, in that

$$
\left.\left[q_{+} j_{i-}(p, q)+q_{-} j_{i+}(p, q)\right]\right|_{F_{i} \text { term }}=\gamma_{-} F_{i}\left(z, Q^{2}\right)\left\{q_{+}-q_{-} \frac{q_{+}}{q_{-}}\right\}=0 .
$$

This result mimics the method for insuring current conservation developed in Ref. [18], and gives some evidence that that method is dynamically sound.

These results are used in the next subsection to calculate the hadronic transition current. 

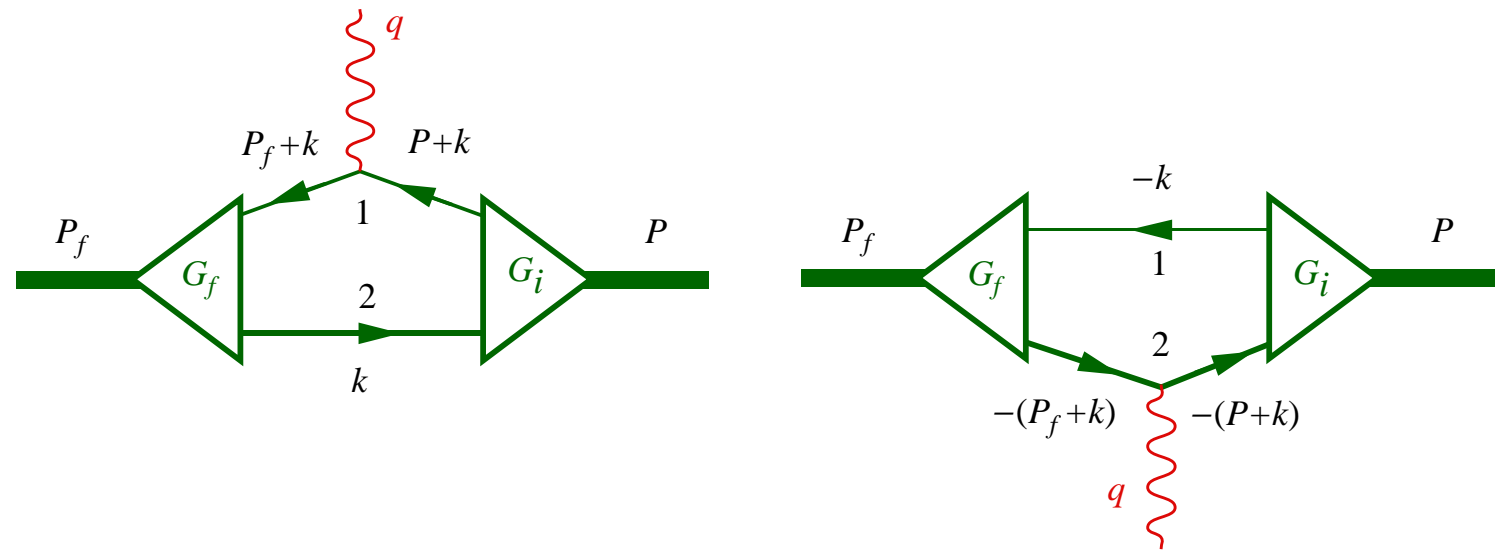

FIG. 10: Currents for the electromagnetic transition from the initial state $i$ to the final state $f$. The left hand diagram is the contribution from the electromagnetic interaction with the quark with mass $m_{1}$ and charge $e_{1}$ and the right hand diagram is the same for the antiquark with mass $m_{2}$ and charge $e_{2}$.

\section{B. The transition current}

In the presence of confinement, and without any meson decay mechanisms, the quarks in a $q \bar{q}$ bound state must remain bound, even after they absorb an energetic photon. In this section we calculate the transition current for the process $\gamma^{*}+M_{i} \rightarrow M_{f}$, where $M_{f}$ is the mass of a (possibly excited) final bound state and $M_{i}$ is the mass of the initial (ground) state.

The transition current consists of the sum of two contributions: one in which the photon is absorbed by the quark with dressed mass $m_{1}$, and a second in which the photon is absorbed by the antiquark with mass $m_{2}$. The momentum used to label each of these processes are shown in Fig. 10, the initial bound state has momentum $P$ and the final state has momentum $P_{f}=P+q$. The transition current coming from the first term is

$$
\begin{aligned}
&\left.\left\langle f\left|\mathcal{J}^{\mu}\left(P_{f}, P\right)\right| i\right\rangle\right|_{e_{1} \text { term }}=i e_{1} N_{c} \int \frac{d^{2} k}{(2 \pi)^{2}} G_{f}\left(-P_{f}-k,-k\right) G_{i}(P+k, k) \\
& \times \operatorname{Tr}\left[\gamma_{-}\left\{\begin{array}{c}
1 \\
\gamma_{x}
\end{array}\right\} S_{1}\left(P_{f}+k\right) j_{1}^{\mu} S_{1}(P+k) \gamma_{-} S_{2}(k)\right] \\
&=i \int \frac{d^{2} k}{(2 \pi)^{2}} \frac{e_{1} N_{c} G_{f}\left(-P_{f}-k,-k\right) G_{i}(P+k, k) N^{\mu}}{\left[m_{1}^{2}-\left(P_{f}+k\right)^{2}-i \epsilon\right]\left[m_{1}^{2}-(P+k)^{2}-i \epsilon\right]\left[m_{2}^{2}-k^{2}-i \epsilon\right]}
\end{aligned}
$$

where $j_{1}^{\mu}$ is the quark current operator for quark 1, as worked out in subsection ஹI A $N^{\mu}$ is the spin dependent numerator discussed below, and we have allowed for the possibility that the final state may have either the $\gamma_{-}$or the $\gamma_{-} \gamma_{x}$ structure discussed above (we will not consider the $\gamma_{-} \gamma_{y}$ states; they are needed only if we consider the $y$ component of the transverse current and give results identical to the results for the $x$ components). Note the factor of $N_{c}$ coming from the sum over all possible quark colors.

Only the term in the numerator of the $S_{2}$ propagator equal to $k_{-} \gamma_{+}$can give a nonzero result, but the terms that contribute from the $S_{1}$ propagators depend on the matrix form of the final state, and of $j_{1}^{\mu}$. Because the trace of a product of gamma matrices with only one factor of $\gamma_{x}$ or $\gamma_{y}$ is zero, the transverse currents (with $\gamma_{x}$ or $\gamma_{y}$ ) will be zero unless the final state also includes $\gamma_{x}$ (or $\gamma_{y}$ for the $y$ component of the current). Hence we have the rules: 
(i) $x$-type transverse currents couple $\gamma_{-}$states to $\gamma_{-} \gamma_{x}$ states only, and (ii) longitudinal currents couple $\gamma_{-}$states to each other. Because of the exact degeneracy of these states, the results in both of these cases use the same momentum-space wave functions.

The integral (3.13) is evaluated in Appendix [F] The result for the - component of the current is

$$
\begin{aligned}
\left.\left\langle f_{-}\left|\mathcal{J}_{-}\left(P_{f}, P\right)\right| i_{-}\right\rangle\right|_{e_{1} \text { term }}=\frac{16 e_{1} N_{c} P_{-}}{(2 \pi) \mathcal{N}^{2}} & \left\{\int_{y}^{1}+\int_{0}^{y} \mathcal{R}\right\} d \xi \Phi_{f}\left(\xi^{\prime}, P_{f}\right) \Phi_{i}(\xi, P) \\
\times & {\left[1+F_{1}\left(\eta, Q^{2}\right)\right] }
\end{aligned}
$$

where

$$
y=-\frac{q_{-}}{P_{-}}, \quad \xi^{\prime}=\frac{\xi-y}{1-y}, \quad \eta=\frac{y-\xi}{y}
$$

[for definitions of all the momentum fractions see Eqs. (F1) and (F2)], the state $f_{-}$has the same structure as the initial state (assumed to be $\gamma_{-}$), $F_{1}$ is the quark form factor defined in Eq. (3.8) and $\mathcal{R}$ is given in Eq. (F14). The plus and transverse components of the current are

$$
\begin{aligned}
\left.\left\langle f_{x}\left|\mathcal{J}_{x}\left(P_{f}, P\right)\right| i_{-}\right\rangle\right|_{e_{1} \text { term }}= & \frac{16 e_{1} N_{c} P_{-}}{(2 \pi) \mathcal{N}^{2}}\left\{\int_{y}^{1}+\int_{0}^{y} \mathcal{R}\right\} d \xi \Phi_{f}\left(\xi^{\prime}, P_{f}\right) \Phi_{i}(\xi, P) \\
& \times \frac{q_{+}\left[m_{1}^{2}-\eta(1-\eta) Q^{2} F_{1}\left(\eta, Q^{2}\right)\right]}{m_{01} \eta(1-\eta) Q^{2}} \\
\left.\left\langle f_{-}\left|\mathcal{J}_{+}\left(P_{f}, P\right)\right| i_{-}\right\rangle\right|_{e_{1} \text { term }}=\frac{16 e_{1} N_{c} P_{-}}{(2 \pi) \mathcal{N}^{2}} & \left\{\int_{y}^{1}+\int_{0}^{y} \mathcal{R}\right\} d \xi \Phi_{f}\left(\xi^{\prime}, P_{f}\right) \Phi_{i}(\xi, P) \\
\times & \frac{q_{+}\left[m_{1}^{2}-\eta(1-\eta) Q^{2} F_{1}\left(\eta, Q^{2}\right)\right]}{q_{-} \eta(1-\eta) Q^{2}}
\end{aligned}
$$

where the state $f_{x}$ has the structure $\gamma_{-} \gamma_{x}$. These results are used in the next subsection, and in the discussion of DIS.

Using the definitions of the momenta shown in Fig. 10, it is easy to see that the second term in the transition current can be obtained from the first by letting $1 \leftrightarrow 2$, as discussed in Appendix [F. The exact expression for the $x$ component of the transition current, which is the sum of the $e_{1}$ and $e_{2}$ contributions, can therefore be written

$$
\left\langle f_{x}\left|\mathcal{J}_{x}\left(P_{f}, P\right)\right| i_{-}\right\rangle=\mathcal{J}_{f i}^{1, x}\left(Q^{2}, y\right)+\mathcal{J}_{f i}^{2, x}\left(Q^{2}, y\right)
$$

where the reduced current $\mathcal{J}_{f i}^{j, x}\left(Q^{2}, y\right)$ is

$$
\mathcal{J}_{f i}^{j, x}\left(Q^{2}, y\right)=\frac{e_{j} m_{0 j} y}{1-y}\left\{\int_{y}^{1}+\int_{0}^{y} \mathcal{R}_{j}\right\} d \xi \frac{\Phi_{f}\left(\xi_{j}^{\prime}, P_{f}\right) \Phi_{i}\left(\xi_{j}, P\right)}{\xi_{j}^{\prime} \xi_{j}} F_{j}^{\mathrm{eff}}\left(\eta_{j}, Q^{2}\right)
$$

with $\left\{\xi_{1}, \xi_{1}^{\prime}, \eta_{1}\right\}=\left\{\xi, \xi^{\prime} \eta\right\}$ and $\left\{\xi_{2}, \xi_{2}^{\prime}, \eta_{2}\right\}=\left\{1-\xi, 1-\xi^{\prime}, 1-\eta\right\}$ and

$$
F_{i}^{\mathrm{eff}}\left(\eta, Q^{2}\right)=\frac{1}{m_{0 i}^{2}}\left[m_{i}^{2}-\eta(1-\eta) Q^{2} F_{i}\left(\eta, Q^{2}\right)\right]
$$

Finally, the function $\mathcal{R}_{1}=\mathcal{R}\left(m_{1}, m_{2}\right)$, was defined in Eq. (F14); the substitutions that convert the $e_{1}$ term into the $e_{2}$ term therefore give $\mathcal{R}_{2}=\mathcal{R}\left(m_{2}, m_{1}\right)$. 


\section{Elastic scattering and normalization of the wave function}

If the scattering is elastic, $x=1$ and the momentum fraction $y=-q_{-} / P_{-}$must be computed from the exact expressions for $q$ and $P$ in the c.m. frame [14]. The result, for elastic scattering from the state $n$, is

$$
y=\frac{Q^{2}+Q \sqrt{4 M_{n}^{2}+Q^{2}}}{2 M_{n}^{2}+Q^{2}+Q \sqrt{4 M_{n}^{2}+Q^{2}}}
$$

As $Q^{2} \rightarrow 0, y \rightarrow 0$ and $\eta$ [defined in Eq. (3.15)] approaches $-\infty, F_{i}\left(\eta, Q^{2}\right) \rightarrow 0$, and the exact result for the elastic curent becomes

$$
\left.\left\langle n\left|\mathcal{J}_{-}(P, P)\right| n\right\rangle\right|_{e_{1} \text { term }}=\frac{16 e_{1} N_{c} P_{-}}{(2 \pi) \mathcal{N}^{2}} \int_{0}^{1} d \xi\left[\Phi_{n}(\xi, P)\right]^{2}=2 e_{1} P_{-} \mathcal{F}_{n}(0),
$$

where charge normalization requires that the form factor $\mathcal{F}_{n}\left(Q^{2}\right)$ be unity at $Q^{2}=0$. Hence

$$
\mathcal{N}=2 \sqrt{\frac{N_{c}}{\pi}},
$$

gives the normalization condition (2.40).

Furthermore, elastic scattering requires that, as $Q^{2} \rightarrow 0$,

$$
(\eta-1) q_{-}=\xi P_{-} \rightarrow \eta q_{-},
$$

and hence

$$
\begin{aligned}
\left.\left\langle n\left|\mathcal{J}_{+}(P, P)\right| n\right\rangle\right|_{e_{1} \text { term }} & =2 e_{1} P_{+} \frac{m_{1}^{2}}{M_{n}^{2}} \int_{0}^{1} d \xi \frac{\Phi_{n}^{2}\left(\xi, M_{0}^{2}\right)}{\xi^{2}} \\
& =2 e_{1} P_{+} \mathcal{F}_{n}(0)=2 e_{1} P_{+},
\end{aligned}
$$

which follows from the wave function identity $(\mathbb{B} 4)$. Note that the charge is properly normalized and conserved for both components because we used the correct dressed quark current (3.6).

Finally, we point out that the elastic transverse current is zero for all $Q^{2}$, as required by the summetries of the states

$$
\left\langle n\left|\mathcal{J}_{x}\left(P_{f}, P\right)\right| n\right\rangle=0 .
$$

We now turn to a discussion of deep inelastic scattering.

\section{DEEP INELASTIC SCATTERING}

\section{A. Extracting the structure functions from the DIS cross section}

Using the standard conventions (also defined in Ref. 14]), the DIS cross section is

$$
\begin{aligned}
\frac{d^{2} \sigma}{d \Omega^{\prime} d E^{\prime}} & =\left(\frac{2 \alpha E^{\prime}}{Q^{2}}\right)^{2}\left[W_{2} \cos ^{2}\left(\frac{\theta}{2}\right)+2 W_{1} \sin ^{2}\left(\frac{\theta}{2}\right)\right] \\
& =\sigma_{M}\left[W_{2}+2 W_{1} \tan ^{2}\left(\frac{\theta}{2}\right)\right],
\end{aligned}
$$


where the structure functions $W_{1}$ and $W_{2}$ are part of the hadronic tensor, defined by

$$
\begin{aligned}
\frac{W_{\mu \nu}}{4 \pi M} & =-\left(g_{\mu \nu}-\frac{q_{\mu} q_{\nu}}{q^{2}}\right) W_{1}+\left(P_{\mu}-q_{\mu} \frac{P \cdot q}{q^{2}}\right)\left(P_{\nu}-q_{\nu} \frac{P \cdot q}{q^{2}}\right) \frac{W_{2}}{M^{2}} \\
& =-\left(g_{\mu \nu}-\frac{q_{\mu} q_{\nu}}{q^{2}}\right) W_{1}+\left(P_{\mu}+\frac{q_{\mu}}{2 x}\right)\left(P_{\nu}+\frac{q_{\nu}}{2 x}\right) \frac{W_{2}}{M^{2}}
\end{aligned}
$$

where $M=M_{1}$ is the target, or ground state mass. The structure function $W_{1}$ is immediately extracted from the $x x$ (or $y y$ ) component of the tensor

$$
M W_{1}=\frac{W_{x x}}{4 \pi} \rightarrow F_{1}(x)=\frac{1}{2} f(x) .
$$

Here we anticipate that $M W_{1}$ scales in the deep inelastic limit to the function $F_{1}(x)$ of the variable $x=Q^{2} /(2 P \cdot q)$. Note that $W_{1}$ must be identically zero if the $x$ (and $y$ ) components of the current are zero.

The structure function $W_{2}$ can be extracted from the + and - components of the current using

$$
W_{-+}=\frac{1}{2}\left(W_{00}-W_{z z}\right)=2 \pi M\left\{W_{2}\left(1+\frac{Q^{2}}{4 x^{2} M^{2}}\right)-W_{1}\right\} .
$$

In the deep inelastic limit this gives

$$
\nu W_{2} \rightarrow F_{2}(x)=2 x F_{1}(x)+\frac{1}{\pi} x W_{-+} .
$$

The contribution from $W_{-+}$will be shown to go like $\nu^{-1}$ in the following sections. Hence, if $F_{1}$ is non-zero, we obtain the Callan-Gross relation. However, if the transverse components of the current are omitted, $F_{1}=0$ and $\nu W_{2}$ does not scale. In this case it is the quanity $\nu^{2} W_{2}$ that scales, and we recover the results of Einhorn [3].

We now turn to a calculation of the structure functions. We calculate them first (i) in the partonic picture, where all final state interations are ignored and the quarks in the final state are assumed to be free, and then (ii) in the hadronic picture, where confinement ensures that the only possible final states are the bound $q \bar{q}$ states we have already discussed. We emphasize that the partonic picture can never actually occur because the confining interaction, which also acts in the final state, can never be ignored.

\section{B. DIS in the partonic picture}

Consider deep inelastic scattering (DIS) in the partonic picture, which assumes a final state composed of free quarks with no interaction. This picture is illustrated in Fig. 11. The full result is the sum of a contributions in which the photon is absorbed by the quark with charge $e_{1}$, and one in which it is absorbed by the antiquark with charge $e_{2}$. In the center of mass of the ejectiles, either particle can go forwards or backwards and the unpolarized cross section is the sum over both helicities of the particles.

Begin by calculating the transverse component (chosen be be $x$ ) of the current. The outgoing quark has a mass of $m_{1}$ and momentum $p_{1}$ in the center of mass frame of the 


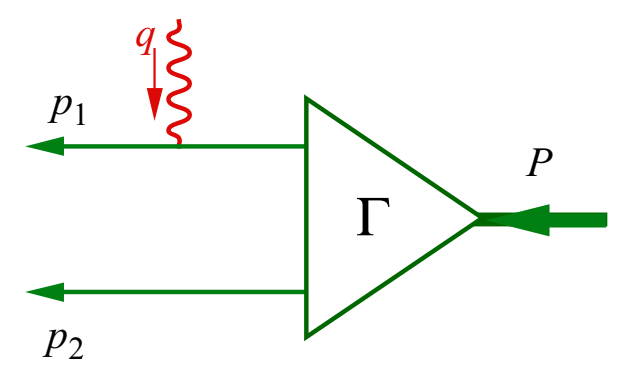

FIG. 11: One of two diagrams that contribute to DIS in the partonic picture. Note the labeling of the momenta.

ejectiles, while the antiquark has a mass of $m_{2}$ and momentum of $p_{2}$ (in this section $p_{2}$ will be the physical momentum of the outgoing antiquark, opposite in sign to that used in the earlier sections of this paper). The current is the sum of two diagrams, only one of which is shown in Fig. 11] Since the $q \bar{q}$ vertex contains the factor of $\gamma_{-}$, only the factor $m+p_{-} \gamma_{+}$in the numerator of the dressed propagator (2.30) survives, but we will retain only the factor of $p_{-} \gamma_{+}$which dominates the result at large $Q^{2}$. The following result is obtained for the $x$ component of the current for the disintegration of the ground state (the subscript $n=1$ usually attached to the ground state will be omitted in this section):

$$
\begin{aligned}
J_{x}= & -e_{1}\left[\bar{u}\left(p_{1}\right) \gamma_{x} \gamma_{+} \gamma_{-} v\left(p_{2}\right)\right] \frac{\left(p_{1}-q\right)_{-} G\left(p_{1}-q,-p_{2}\right)}{m_{1}^{2}-\left(p_{1}-q\right)^{2}} \\
& +e_{2}\left[\bar{u}\left(p_{1}\right) \gamma_{-} \gamma_{+} \gamma_{x} v\left(p_{2}\right)\right] \frac{\left(p_{2}-q\right)_{-} G\left(p_{1},-p_{2}+q\right)}{m_{2}^{2}-\left(p_{2}-q\right)^{2}}
\end{aligned}
$$

Introducing the momentum of the bound state, $P=p_{1}+p_{2}-q$, and defining

$$
z=\frac{\left(p_{1}-q\right)_{-}}{P_{-}}=\frac{\left(P-p_{2}\right)_{-}}{P_{-}}
$$

[as in Eq. (2.36)], the first denominator can be written

$$
\begin{aligned}
m_{1}^{2}-\left(p_{1}-q\right)^{2} & =m_{1}^{2}-\left(P-p_{2}\right)^{2}=m_{1}^{2}-2\left[\frac{M^{2}}{2 P_{-}}-\frac{m_{2}^{2}}{2 p_{2-}}\right]\left(P-p_{2}\right)_{-} \\
& =m_{1}^{2}-z\left(M^{2}-\frac{m_{2}^{2}}{1-z}\right)=z\left[\frac{m_{1}^{2}}{z}+\frac{m_{2}^{2}}{1-z}-M^{2}\right] .
\end{aligned}
$$

Here we have made use of the constraints $M^{2}=2 P_{-} P_{+}$and $m_{2}^{2}=2 p_{2-} p_{2+}$. A similar result follows for the second denominator if we define a new momentum fraction $z^{\prime}$

$$
z^{\prime}=\frac{\left(p_{2}-q\right)_{-}}{P_{-}}=\frac{\left(P-p_{1}\right)_{-}}{P_{-}} .
$$

With these definitions we obtain

$$
J_{x}=-e_{1}\left[\bar{u}\left(p_{1}\right) \gamma_{x} \gamma_{+} \gamma_{-} v\left(p_{2}\right)\right] \frac{P_{-} G(z, P)}{\Delta\left(z, M^{2}\right)}
$$




$$
\begin{aligned}
& +e_{2}\left[\bar{u}\left(p_{1}\right) \gamma_{-} \gamma_{+} \gamma_{x} v\left(p_{2}\right)\right] \frac{P_{-} G\left(1-z^{\prime}, P\right)}{\Delta\left(1-z^{\prime}, M^{2}\right)} \\
= & -\frac{e_{1}}{\mathcal{N}}\left[\bar{u}\left(p_{1}\right) \gamma_{x} \gamma_{+} \gamma_{-} v\left(p_{2}\right)\right] \Phi(z, P) \\
& +\frac{e_{2}}{\mathcal{N}}\left[\bar{u}\left(p_{1}\right) \gamma_{-} \gamma_{+} \gamma_{x} v\left(p_{2}\right)\right] \Phi\left(1-z^{\prime}, P\right),
\end{aligned}
$$

where the answer has been expressed in terms of the wave function (2.39).

The current and the DIS cross section are evaluated in the c.m. frame, using the kinematics defined in Appendix E. The unpolarized cross section is obtained by squaring the current and summing over spins using

$$
\begin{aligned}
\sum_{\text {spins }} \bar{u}\left(p_{1}\right) \gamma_{x} \gamma_{+} \gamma_{-} v\left(p_{2}\right) \bar{v}\left(p_{2}\right) \gamma_{-} \gamma_{+} \gamma_{x} u\left(p_{1}\right) & =p_{1+} p_{2-} \operatorname{trace}\left[\gamma_{+} \gamma_{-} \gamma_{+} \gamma_{x} \gamma_{-} \gamma_{x} \gamma_{+} \gamma_{-}\right] \\
& =32 p_{1+} p_{2-},
\end{aligned}
$$

and neglecting the interference term (which vanishes in the large $Q^{2}$ limit). Observing that $z \rightarrow z^{\prime} \rightarrow x$ in the DIS limit, and summing over all colors of the outgoing quarks (which do not interfere) gives

$$
\begin{aligned}
\sum_{\text {spins }}\left|J_{x}\right|^{2} & =\frac{32 N_{c}}{\mathcal{N}^{2}}\left\{e_{1}^{2} p_{1+}^{(1)} p_{2-}^{(1)} \Phi^{2}(x, P)+e_{2}^{2} p_{1-}^{(2)} p_{2+}^{(2)} \Phi^{2}(1-x, P)\right\} \\
& =4 \pi Q^{2}\left(\frac{1-x}{x}\right)\left\{e_{1}^{2} \Phi^{2}(x, P)+e_{2}^{2} \Phi^{2}(1-x, P)\right\},
\end{aligned}
$$

where the momentum are evaluated in Appendix E. The hadronic tensor defined in Eq. (4.2) is related to the square of the currents by

$$
\frac{W_{\mu \nu}}{4 \pi M}=\int \frac{d p_{1} d p_{2}}{(2 \pi)^{2} 4 p_{10} p_{20}}(2 \pi)^{2} \delta^{2}\left(P_{f}-P-q\right) \sum_{\mathrm{spins}} J_{\mu} J_{\nu}
$$

The $W_{x x}$ component of the tensor is [14] is therefore

$$
\begin{aligned}
W_{x x} & =4 \pi M W_{1}=\frac{1}{4\left|p_{z}\right|\left(p_{10}+p_{20}\right)} \sum_{\text {spins }}\left|J_{x}\right|^{2} \\
& =2 \pi\left\{e_{1}^{2} \Phi^{2}(x, P)+e_{2}^{2} \Phi^{2}(1-x, P)\right\} .
\end{aligned}
$$

This gives the familiar parton model result for the structure function $W_{1}$

$$
M W_{1}=F_{1}(x)=\frac{1}{2}\left\{e_{1}^{2} \Phi^{2}(x, P)+e_{2}^{2} \Phi^{2}(1-x, P)\right\}=\frac{1}{2} f(x) .
$$

The structure function $W_{2}$, given in Eq. (4.5), is then

$$
\nu W_{2}=2 M x W_{1}=2 x F_{1}(x)=F_{2}(x)=x f(x),
$$

where we anticipate the result $W_{-+} \rightarrow 0$ as $Q^{2} \rightarrow \infty$. This is the Callan-Gross relation.

Now look at the plus and minus components of the current. If the Dirac space were restricted to two dimensions, only these components of the current would exist. The minus component can be obtained immediately from (4.10) by replacing $\gamma_{x}$ with $\gamma_{-}$, giving

$$
J_{-}=-\left[\bar{u}\left(p_{1}\right) \gamma_{-} v\left(p_{2}\right)\right] \frac{2}{\mathcal{N}}\left\{e_{1} \Phi(z, P)-e_{2} \Phi\left(1-z^{\prime}, P\right)\right\}
$$


The plus component could include the two contributions from the quark current, Eq. (3.6), but in the partonic picture the outgoing quark is not dressed, so the second part of the current proportional to $g^{2}$ should be ignored. However, in order to study the contribution of this term we will retain it for now. The leading contribution from both terms in the $J_{+}$ current is

$$
\begin{aligned}
J_{+} & =-\frac{e_{1}}{\mathcal{N}}\left\{m_{01} \frac{\Phi(z, P)}{p_{1-}^{(1)}-q_{-}}\left[\bar{u}\left(p_{1}\right) \gamma_{+} \gamma_{-} v\left(p_{2}\right)\right]+\frac{g^{2}}{\pi} \frac{\Phi(z, P)}{p_{1-}^{(1)}\left(p_{1-}^{(1)}-q_{-}\right)}\left[\bar{u}\left(p_{1}\right) \gamma_{-} v\left(p_{2}\right)\right]\right\} \\
& +\frac{e_{2}}{\mathcal{N}}\left\{m_{02} \frac{\Phi\left(1-z^{\prime}, P\right)}{p_{2-}^{(2)}-q_{-}}\left[\bar{u}\left(p_{1}\right) \gamma_{-} \gamma_{+} v\left(p_{2}\right)\right]+\frac{g^{2}}{\pi} \frac{\Phi(z, P)}{p_{2-}^{(2)}\left(p_{2-}^{(2)}-q_{-}\right)}\left[\bar{u}\left(p_{1}\right) \gamma_{-} v\left(p_{2}\right)\right]\right\} .
\end{aligned}
$$

The $W_{-+}$component of the hadron tensor is then

$$
W_{-+}=\frac{1}{4\left|p_{z}\right|\left(p_{10}+p_{20}\right)} \sum_{\text {spins }} J_{-} J_{+}=\frac{x}{2 Q^{2}(1-x)} \sum_{\text {spins }} J_{-} J_{+} .
$$

Using (4.17) and (4.18), and summing over colors, the $e_{1}^{2}$ contribution to the current sum becomes, at large $Q^{2}$,

$$
\begin{aligned}
\left.\sum_{\text {spins }} J_{-} J_{+}\right|_{e_{1} \text { term }} & =\frac{32 e_{1}^{2} N_{c}}{\mathcal{N}^{2}} \Phi^{2}(x, P)\left[\frac{p_{2-}^{(1)}}{p_{1-}^{(1)}-q_{-}}\right]\left(m_{01}^{2}-\frac{g^{2}}{\pi}\right) \\
& =8 \pi e_{1}^{2} m_{1}^{2}\left(\frac{1-x}{x}\right) \Phi^{2}(x, P) .
\end{aligned}
$$

Hence

$$
\left.W_{-+}\right|_{e_{1} \text { term }}=\frac{4 \pi e_{1}^{2}}{Q^{2}} m_{1}^{2} \Phi^{2}(x, P)=\left.4 \pi \frac{m_{1}^{2}}{Q^{2}} f(x)\right|_{e_{1} \text { term }}
$$

and this term is subleading at high $Q^{2}$. Note that the effect of neglecting the term proportional to $g^{2}$ in the + component of the quark current (as we are instructed to do in the partonic picture) is to replace $m_{1}^{2}$ by the bare quark mass $m_{01}^{2}$. If $W_{1}=0$, then $\nu^{2} W_{2}$ scales as

$$
\left.\left.\nu^{2} W_{2}\right|_{e_{1} \text { term }} \rightarrow \frac{Q^{2}}{2 \pi M} W_{-+}\right|_{e_{1} \text { term }}=\left.2 \frac{m_{01}^{2}}{M} f(x)\right|_{e_{1} \text { term }}
$$

Up to a factor, this is the result originally obtained by Einhorn [3]. In that paper, the Dirac space was restricted to 2 dimensions, $W_{1}$ was necessarily zero, and $\nu^{2} W_{2}$ scaled as (4.22). This distribution amplitude does not have the correct physical interpretation. In particular, it predicts that the DIS cross section depends on the square of bare quark mass, a clearly unphysical result. Only by considering the full four dimensional Dirac space are we able to obtain scaling for $\nu W_{2}$, and the familiar physical result (4.15) for $f(x)$.

Another consequence of the fact that $W_{-+}$is subleading is that we can avoid coming to grips with the fact that the current components $J_{-}$and $J_{+}$, as defined above, do not conserve current. Since these components do not contribute to the final result, we need not discuss how they can be redefined in order to conserve current. 


\section{DIS in the hadronic picture}

\section{Exact results for inelastic scattering}

The exact hadronic tensor (4.2) for electroproduction of a single final state $f$ with mass $M_{n}$ (denoted by $n$ ) from the ground state $i$ with mass $M_{1} \equiv M$ (denoted by 0 ) is

$$
\begin{aligned}
\left.W_{\mu \nu}\right|_{f} & =\int \frac{d P_{f z}}{(2 \pi) 2 P_{f 0}}(2 \pi)^{2} \delta^{2}\left(P_{f}-P-q\right) \sum_{\text {spins }}\left\langle 0\left|\mathcal{J}_{\mu}\right| n\right\rangle\left\langle n\left|\mathcal{J}_{\nu}\right| 0\right\rangle \\
& =\delta\left(M_{n}-P_{0}-q_{0}\right) \frac{\pi}{M_{n}} \sum_{\text {spins }}\left\langle 0\left|\mathcal{J}_{\mu}\right| n\right\rangle\left\langle n\left|\mathcal{J}_{\nu}\right| 0\right\rangle
\end{aligned}
$$

This is a delta function. In any physical measurement, the detectors will accept a finite range of values of the final electron energy, $E^{\prime}$, requiring that this theoretical cross section be averaged over $E^{\prime}$. This averaging process will be discussed in subsection IVD below, where the deta function will be written as a delta function in the Bjorken variable $x$, which spikes at values of $x_{n}$ corresponding to the excitation of the final state $n$. In the c.m. system, this leads to the following exact transformation

$$
\delta\left(M_{n}-P_{0}-q_{0}\right)=\delta\left(x-x_{n}\right) \frac{2 x_{n}^{2} M_{n}}{Q^{2}}
$$

with $x_{n}$ the value of $x$ at the bound state mass $M_{n}$, given in Eq. (4.26) below.

In the DIS limit, the transverse currents dominate the scattering. The exact result for the transverse tensor, $W_{x x}$, that describes the scattering from the ground state with $\gamma_{-}$ structure, to a single transverse final state $n$ with $\gamma_{-} \gamma_{x}$ structure, is

$$
\left.W_{x x}\right|_{n}=\delta\left(x-x_{n}\right) \frac{2 \pi x_{n}^{2}}{Q^{2}}\left\{\mathcal{J}_{n}^{1, x}\left(Q^{2}, y\right)+\mathcal{J}_{n}^{2, x}\left(Q^{2}, y\right)\right\}^{2}
$$

where the reduced transverse current was defined in Eq. (3.18). (Here the $f i$ subscript is replaced by $n$, and denotes a transition between the ground and $n$th state.) Next we observe that the value of $x$ at which the final state is excited is

$$
x_{n}=\frac{Q^{2}}{M_{n}^{2}-M^{2}+Q^{2}},
$$

and that the spacing between neighboring levels is

$$
\Delta_{x_{n}}=x_{n}-x_{n+1}=\frac{\delta M_{n}^{2}}{Q^{2}} x_{n} x_{n+1},
$$

with $\delta M_{n}^{2}=M_{n+1}^{2}-M_{n}^{2}$. Hence the exact result for the total inelastic tensor $W_{x x}$ is

$$
W_{x x}=\sum_{n} \delta\left(x-x_{n}\right) \Delta_{x_{n}}\left(\frac{2 \pi}{\delta M_{n}^{2}}\right)\left(\frac{x_{n}}{x_{n+1}}\right)\left\{\mathcal{J}_{n}^{1, x}\left(Q^{2}, y\right)+\mathcal{J}_{n}^{2, x}\left(Q^{2}, y\right)\right\}^{2},
$$

where the sum is over all possible final states $n$. 


\section{Hadronic result in the DIS limit}

The DIS limit is defined by the requirement that $Q^{2} \rightarrow \infty$ with $x$ held constant. In this limit both $Q^{2}$ and $M_{n}^{2}$ become large, with

$$
x_{n} \rightarrow \frac{Q^{2}}{M_{n}^{2}+Q^{2}}, \quad\left(\text { as } Q^{2} \text { and } M_{n}^{2} \rightarrow \infty\right) .
$$

Study of the DIS limit therefore requires estimating the transition form factors for large final state number $n$, but for all $x$ (since $x_{n}$ depends on $Q^{2}$ ). In the DIS limit, $y \rightarrow x$ [easily seen from the expansions (E1)], so $y$ will be replaced by $x$ in the following discussion.

When $n$ is large the "tails" of the wave functions are very small, and the exact transition current (3.18) can be approximated by neglecting the contribution from the region $\xi \in[0, x]$ (where $\xi^{\prime}<0$ ), giving

$$
\begin{aligned}
\mathcal{J}_{n}^{1, x}\left(Q^{2}, x\right) & \simeq \frac{e_{1} m_{01} x}{1-x} \int_{y}^{1} d \xi \frac{\Phi_{n}\left(\xi^{\prime}, P_{f}\right) \Phi(\xi, P)}{\xi^{\prime} \xi} F_{1}^{\mathrm{eff}}\left(\eta, Q^{2}\right) \\
& =e_{1} m_{01} x \int_{0}^{1} d \xi^{\prime} \frac{\Phi_{n}\left(\xi^{\prime}, P_{f}\right) \Phi\left(\left[\xi^{\prime}(1-x)+x\right], P\right)}{\xi^{\prime}\left[\xi^{\prime}(1-x)+x\right]} F_{1}^{\mathrm{eff}}\left(\eta, Q^{2}\right),
\end{aligned}
$$

where we continue to suppress the label $n=1$ on the ground (initial) state wave function, and

$$
\eta=-\xi^{\prime}\left(\frac{1-x}{x}\right)<0
$$

in the region of integration. When $\eta<0$ and $Q^{2} \rightarrow \infty$, the relation (D10) may be used to approximate the quark form factor by

$$
F_{i}\left(\eta, Q^{2}\right) \rightarrow-\frac{g^{2}}{\pi Q^{2} \eta(1-\eta)}
$$

giving

$$
F_{1}^{\mathrm{eff}}\left(\eta, Q^{2}\right) \rightarrow 1
$$

Equation (4.30) is now further reduced by expanding $\Phi(\xi, P) / \xi$ around $\xi=x$

$$
\frac{\Phi(\xi, P)}{\xi}=\frac{\Phi(x, P)}{x}+\delta \frac{d}{d x}\left(\frac{\Phi(x, P)}{x}\right)+\frac{1}{2} \delta^{2} \frac{d^{2}}{d x^{2}}\left(\frac{\Phi(x, P)}{x}\right)+\cdots
$$

where the higher order terms are proportional to powers of $\delta$

$$
\delta \equiv \xi^{\prime}(1-x)
$$

This replacement gives the following series for $\mathcal{J}$

$$
\begin{aligned}
\mathcal{J}_{n}^{1, x}\left(Q^{2}, x\right)= & e_{1} m_{01} \Phi(x, P) \int_{0}^{1} d \xi^{\prime} \frac{\Phi_{n}\left(\xi^{\prime}, P_{f}\right)}{\xi^{\prime}} \\
& +e_{1} m_{01} \sum_{m=1}^{m=\infty} d_{n}^{m} \frac{(1-x)^{m}}{m !}\left(\frac{d}{d x}\right)^{m}\left(\frac{\Phi(x, P)}{x}\right)
\end{aligned}
$$


where

$$
d_{n}^{m}=\int_{0}^{1} d \xi^{\prime} \xi^{\prime m-1} \Phi_{n}\left(\xi^{\prime}, P_{f}\right)
$$

Using the identity (B10) and the other estimates given in Appendix B it follows that

$$
\lim _{n \rightarrow \infty} \frac{d_{n}^{m}}{C_{n}} \rightarrow \frac{\text { const }}{n} \rightarrow 0
$$

Hence the large $n$ approximation to (4.36) is

$$
\begin{aligned}
\mathcal{J}_{n}^{1, x}\left(Q^{2}, x\right) & \rightarrow e_{1} m_{01} \Phi(x, P) \int_{0}^{1} d \xi^{\prime} \frac{\Phi_{n}\left(\xi^{\prime}, P_{f}\right)}{\xi^{\prime}} \\
& =e_{1} C_{n} \Phi(x, P) .
\end{aligned}
$$

The plus component of the transition current is smaller than the transverse current by a factor of $m_{01} / q_{-}$[compare the two results in Eq. (3.16)] , and hence is obtained immediately from the result (4.39). Using Eq. (E1) for $q_{-}$, and Eq. (4.29) to replace $\sqrt{\left(1-x_{n}\right) / x_{n}}$, gives

$$
\mathcal{J}_{n}^{1,+}\left(Q^{2}, x\right) \simeq-\sqrt{2} e_{1} C_{n} \frac{m_{01} M_{n}}{Q^{2}} \Phi(x, P) .
$$

The minus component can also be obtained from Eq. (4.39). Reviewing the derivation, we see that the minus component, in the DIS limit, is obtained from the transverse component by replacing

$$
r \equiv \frac{m_{01}}{2 \eta(1-\eta) q_{-}}=\frac{m_{01} y}{\sqrt{2} \xi^{\prime} \xi M_{n}} \rightarrow 1
$$

Hence, multiplying (4.39) by $r^{-1}$

$$
\begin{aligned}
\mathcal{J}_{n}^{1,-}\left(Q^{2}, x\right) & \simeq \sqrt{2} e_{1} \Phi(x, P)\left\{M_{n} \int_{0}^{1} d \xi^{*} \Phi_{f}\left(\xi^{*}, P_{f}\right)\right\} \\
& \simeq \sqrt{2} e_{1} C_{n}\left(\frac{m_{01}+(-1)^{n-1} m_{02}}{M_{n}}\right) \Phi(x, P),
\end{aligned}
$$

where the $\xi^{\prime}$ integral was evaluated using identity (B11).

The transition currents can be shown to be gauge invariant by useing the Ward-Takahashi identity on the quark-photon vertex, and reducing the result using the Bethe-Salpeter equation.

These currents will now be used to determine the structure functions $W_{1}$ and $W_{2}$ in the hadron picture.

\section{The DIS cross section from the transition form factors}

The cross section in the DIS limit can now be obtained from the exact formula (4.28). As long as $x$ is not too close to unity each term in the sum must correspond to some state with large $n$ (the states with small $n$ "pile up" near $x=1$ where their total contribution 
is very small), and the approximate result for the transverse current, (4.39), may be used. Furthermore, for large $n, \delta M_{n}^{2}$ may be approximated by using (B13), and $x_{n+1} \simeq x_{n}$. Hence the final hadronic result [denoted $\tilde{f}$ to distinguish it from the partonic result $f$ of Eq. (4.15)] in the DIS limit is

$$
\tilde{f}(x)=2 \tilde{F}_{1}(x)=\frac{W_{x x}}{2 \pi}=\sum_{n} \delta\left(x-x_{n}\right) \Delta_{x_{n}}\left\{e_{1} \Phi(x, P)+(-1)^{n} e_{2} \Phi(1-x, P)\right\}^{2} .
$$

Note that $\tilde{F}_{1}(x)$ is a series of separate spikes, zero for all $x$ except at particular values $x_{n}$, where it is infinite. The partonic function, $F_{1}(x)$, is a smooth function of $x$. To compare $\tilde{F}_{1}(x)$ to $F_{1}(x)$, we must average over $x$. This will lead to the concept of duality and will be discussed in the next subsection below.

Now look at the $W_{-+}$component of the tensor. Replacing the transverse components of the current in Eq. (4.28) with plus and minus components, and taking the DIS limit, gives

$$
\begin{aligned}
W_{-+}= & \frac{2 \pi}{C_{\infty}^{2}} \sum_{n} \delta\left(x-x_{n}\right) \Delta_{x_{n}} \\
& \times\left\{\mathcal{J}_{n}^{1,-}\left(Q^{2}, x\right)+\mathcal{J}_{n}^{2,-}\left(Q^{2}, x\right)\right\}\left\{\mathcal{J}_{n}^{1,+}\left(Q^{2}, x\right)+\mathcal{J}_{n}^{2,+}\left(Q^{2}, x\right)\right\}^{*} .
\end{aligned}
$$

Substituting Eqs. (4.40) and (4.42), and droping the term proportional to $(-1)^{n-1}$ (which averages to zero) gives

$$
\left.W_{-+}\right|_{e_{1} \text { term }}=\frac{4 \pi m_{01}^{2}}{Q^{2}} \sum_{n} \delta\left(x-x_{n}\right) \Delta_{x_{n}} e_{1}^{2} \Phi^{2}(x, P) .
$$

This is a nonleading term and can be neglected in the DIS limit. Hence the hadronic picture also gives the Callan-Gross relation, and it is sufficient to compare the functions $F_{1}$ and $\tilde{F}_{1}$ only.

If $W_{1}$ were zero, Eq. (4.45) would give the following scaling relation for $\nu^{2} W_{2}$

$$
\left.\left.\nu^{2} W_{2}\right|_{e_{1} \text { term }} \rightarrow \frac{Q^{2}}{2 \pi M} W_{-+}\right|_{e_{1} \text { term }}=\left.2 \frac{m_{01}^{2}}{M} \tilde{f}(x)\right|_{e_{1} \text { term }}
$$

This is to be compared with (4.22). Hence, the duality of $W_{1}$ and the duality of the nonleading terms in $\nu^{2} W_{2}$ depends on comparison of the same functions, $f(x)$ and $\tilde{f}(x)$.

We discuss this comparison now.

\section{Duality and its implications}

In any physical measurement, the detectors which define the final state will accept a range of final electron energies $\delta E^{\prime}$. For fixed $Q^{2}$, this can be converted into the acceptance of a range of values of $x$ centered at $x_{i}$ with width $\delta x$ so that $x$ varies over the interval bounded by $x_{i \pm}=x_{i} \pm \delta x / 2$. Then the experimentally measured hadronic structure function in the DIS limit can be computed from (4.43)

$$
\begin{aligned}
\langle\tilde{f}\rangle_{x_{i}} & \equiv \frac{1}{\delta x} \int_{x_{i-}}^{x_{i+}} d x \tilde{f}(x) \\
& =\frac{1}{\delta x} \sum_{n \in x_{i}} \Delta_{x_{n}}\left|e_{1} \Phi\left(x_{n}, P\right)+(-1)^{n-1} e_{2} \Phi\left(1-x_{n}, P\right)\right|^{2} .
\end{aligned}
$$




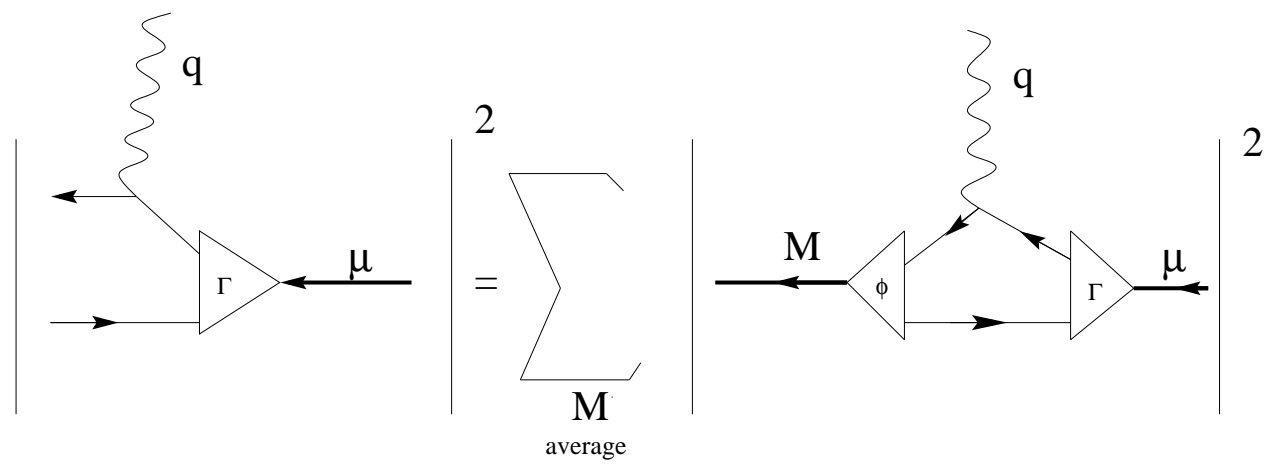

FIG. 12: Quark-hadron duality

Note that this is the sum of smooth terms proportional to $e_{1}^{2}$ and $e_{2}^{2}$, and a rapidly oscillating interference term proportional to $e_{1} e_{2}$. In the DIS limit, the separation $\Delta_{x}$ between states approaches zero, and therefore a large number of states are necessarily included in any interval $\delta x$. The interference term, which changes sign as $(-1)^{n-1}$, therefore averages to zero. This cancellation of the interference term was pointed out originally by Einhorn [3], and empahsized recently by Close and Isgur in the context of the nonrelativistic quark model [19].

The smooth terms can be approximated by their value at the center of the interval. Using the fact that the number of states in the interval times the $x$ spacing between them must necessarily equal the width $\delta x$, so that

$$
\sum_{n \in x_{i}} \Delta_{x_{n}}=\delta x
$$

we can reduce (4.47) to

$$
\begin{aligned}
\langle\tilde{f}\rangle_{x_{i}} \simeq & \left\{e_{1}^{2} \Phi^{2}\left(x_{i}, P\right)+e_{2}^{2} \Phi^{2}\left(1-x_{i}, P\right)\right\} \frac{1}{\delta x} \sum_{n \in x_{i}} \Delta_{x_{n}} \\
& +2 e_{1} e_{2} \frac{1}{\delta x} \sum_{n \in x_{i}}(-1)^{n-1} \Delta_{x_{n}} \Phi\left(x_{n}, P\right) \Phi\left(1-x_{n}, P\right) \\
\rightarrow & e_{1}^{2} \Phi^{2}\left(x_{i}, P\right)+e_{2}^{2} \Phi^{2}\left(1-x_{i}, P\right)=f\left(x_{i}\right)
\end{aligned}
$$

where $f(x)$ was defined in Eq. (4.15).

This result should be compared with the partonic result, averaged in the same way. Since the partonic result is smooth (and the interference term already neglected) the partonic average is trival

$$
\langle f\rangle_{x_{i}} \equiv \frac{1}{\delta x} \int_{x_{i-}}^{x_{i+}} d x f(x) \simeq f\left(x_{i}\right) \frac{1}{\delta x} \int_{x_{i-}}^{x_{i+}} d x=f\left(x_{i}\right)
$$

Hence we see that

$$
\langle\tilde{f}\rangle_{x_{i}}=\langle f\rangle_{x_{i}}
$$

proving duality. 
Note that a similar identity holds for the $n$th moments of $f$. In the high $Q^{2}$ limit when the final states are numerous and close enough together so that $\Delta_{x_{n}} \rightarrow d x \rightarrow 0$, the $m$ th moment of the hadronic structure function is

$$
\begin{aligned}
\left\langle x^{m}\right\rangle \equiv \int_{0}^{1} d x x^{m} \tilde{f}(x) & =\sum_{n} \Delta_{x_{n}} x_{n}^{m}\left[e_{1}^{2} \Phi^{2}\left(x_{n}, P\right)+e_{2}^{2} \Phi^{2}\left(1-x_{n}, P\right)\right] \\
& \rightarrow \int_{0}^{1} d x x^{m}\left[e_{1}^{2} \Phi^{2}(x, P)+e_{2}^{2} \Phi^{2}(1-x, P)\right] \\
& =\int_{0}^{1} d x x^{m} f(x) .
\end{aligned}
$$

Quark-hadron duality means that properly averaged hadronic observables in a certain kinematic regime (high $Q^{2}$ ) can be described by perturbative QCD, as schematically represented in Fig. 12 and demonstrated in Eq. (4.51). Recent data from Jefferson Laboratory exhibit duality, and this has focused attention on this old subject. Recent studies show that the weak decays within the 't Hooft model exhibit duality [11, 12, 13], and in the framework of DIS, Close and Isgur [19] studied its emergence in the context of realistic nonrelativistic quark models. A recent paper by Isgur, Jeschonnek, Melnitchouk and Van Orden [20] studied duality using a relativistic Klein-Gordon equation with a confining interaction. This work is complementary to these other studies.

While we have proved that duality emerges in the DIS limit, and have corrected the pathologies of earlier work, we have not yet studied how duality emerges as a function of $Q^{2}$. A future paper will present numerical studies of the onset of duality for rQCD [15].

\section{CONCLUSIONS}

We have shown that a very satisfactory description of deep inelastic scattering can be obtained from large $N_{c}$ QCD in 1+1 dimensions, provided the quark spin degrees of freedom are extended to the full $1+3$ dimensions (along with the leptons and the electromagnetic field). We refer to this new model as reduced QCD, and suggest that rQCD may be used to provide insight into the behavior of many other physical processes. In the applications to DIS discussed in this paper, we are able to confirm the Callan-Gross relation, and that duality emerges in the deep inelastic limit.

Our theoretical discussion teaches several lessons. Electromagnetic gauge invariance and duality emerge only if the quark current is fully and consistently dressed. The way in which a quark form factor emerges from this dynamical model mimics the phenomenlogical approach of Gross and Riska [18], showing that their prescription has a dynamical justification. Our treatment of confinement removes all singularities, giving a finite mass for the dressed quark. This mass is gauge dependent, showing that it is not a physical quantity, which can be taken as a demonstration of confinement. But the fact that we have finte dressed quark masses in the presence of confinement provides justification for the constituent quark model. The simultaneous presence of confinement and finite quark mass is possible only if the "on-shell" quark scattering amplitude is identicallyt zero, and we have shown that this is indeed true, as modeled previously in Ref. [17].

The treatment of reduced QCD presented here lays a foundation for much further study. 


\section{ACKNOWLEDGMENTS}

This work has been supported in part by the DOE through grant No. DE-FG0588ER40435. The Southeastrn Universities Research Association (SURA) operates the Thomas Jefferson National Accelerator Facility under DOE contract DE-ACO5-84er40150. One of us (Z. B.) has been supported by a Predoctoral Fellowship at the College of William \& Mary and by the Fundação para a Ciência e a Tecnologia under the post doctoral grant $\mathrm{SFRH} / \mathrm{BPD} / 5661 / 2001$ the project grant CFIF-Plurianual.

\section{APPENDIX A: REDUCTION OF THE TWO QUARK INTERACTION}

Integrals over the variable $k_{+}$like the one encountered in Eq. (2.35) appear several places in this paper. Here we evaluate it in full generality.

Consider the integral

$$
I_{2}\left(p_{-}, r\right)=4 i \int \frac{d^{2} k}{(2 \pi)^{2}} \frac{V(p, k) k_{-}\left(k_{-}-r_{-}\right) F\left(k_{-}, r\right)}{\left[m_{1}^{2}-k^{2}-i \epsilon\right]\left[m_{2}^{2}-(k-r)^{2}-i \epsilon\right]} .
$$

Substituting for $V$ gives

$$
I_{2}\left(p_{-}, r\right)=\frac{i g^{2}}{\pi^{2}} \int \frac{d^{2} k}{\left(p_{-}-k_{-}\right)^{2}}\left\{\frac{F\left(k_{-}, r\right)}{d_{2}\left(k_{-}, k_{+}, r\right)}-\frac{F\left(p_{-}, r\right)}{d_{2}\left(p_{-}, k_{+}, r\right)}\right\},
$$

where the denominator $d_{2}$ is

$$
\begin{aligned}
d_{2}\left(k_{-}, k_{+}, r\right) & =\left[\frac{m_{1}^{2}}{k_{-}}-2 k_{+}-i \frac{\epsilon}{k_{-}}\right]\left[\frac{m_{2}^{2}}{(k-r)_{-}}-2(k-r)_{+}-i \frac{\epsilon}{(k-r)_{-}}\right] \\
& =\left[\frac{m_{1}^{2}}{y r_{-}}-2 k_{+}-i \frac{\epsilon}{y r_{-}}\right]\left[-\frac{m_{2}^{2}}{(1-y) r_{-}}-2(k-r)_{+}+i \frac{\epsilon}{(1-y) r_{-}}\right],
\end{aligned}
$$

and we have introduced the momentum fractions (2.36). This shows that the denominator has only two poles in $k_{+}$, and that they will both be in the same half of the complex plane (giving zero for the integral) unless

$$
0 \leq y \leq 1
$$

Doing the integral over $k_{+}$, and expressing the answer in terms of the momentum fractions $y$ and $z$ gives

$$
\begin{aligned}
I_{2}\left(z r_{-}, r\right) \rightarrow I_{2}(z, r)= & \frac{g^{2}}{\pi} \int_{0}^{1} \frac{d y}{(z-y)^{2}}\left\{\frac{F\left(y r_{-}, r\right)}{\Delta\left(y, r^{2}\right)}-\theta(z) \theta(1-z) \frac{F\left(z r_{-}, r\right)}{\Delta\left(z, r^{2}\right)}\right\} \\
& -\theta(z) \theta(1-z) \frac{g^{2} F\left(z r_{-}, r\right)}{\pi \Delta\left(z, r^{2}\right)}\left\{\int_{-\infty}^{0}+\int_{1}^{\infty}\right\} \frac{d y}{(z-y)^{2}},
\end{aligned}
$$

where the new denominator $\Delta\left(y, r^{2}\right)$ is

$$
\Delta\left(y, r^{2}\right)=\left[\frac{m_{1}^{2}}{y}+\frac{m_{2}^{2}}{(1-y)}-r^{2}\right] .
$$


[Note that this result holds for both signs of $r_{-}$. This is because the sign of the countor integral, which depends on the sign of $r_{-}$, is cancelled by the sign of the integral over $k_{-}=y r_{-}$, which must be changed from $\int_{1}^{0} \rightarrow-\int_{0}^{1}$ when $r_{-}<0$.] It is important to note that the integral over $y$ is not restricted in the term in (A5) that is proportional to $F\left(z r_{-}, r\right)$ because for that term the restriction (A4) applies to $z$ and not $y$. This term not only regulates the singularity in the first term (which arises only if $0 \leq z \leq 1$ ), but also contibutes an additional contribution. Furthermore, if $z$ lies outside of the interval $0 \leq z \leq 1$, the first term is non singular (except at the end points of the interval) and is nonzero! Hence, in the region $0 \leq z \leq 1$ the integral (A5) becomes

$$
\begin{aligned}
I_{2}(z, r) & =\frac{g^{2}}{\pi} \int_{0}^{1} \frac{d y}{(z-y)^{2}}\left\{\frac{F\left(y r_{-}, r\right)}{\Delta\left(y, r^{2}\right)}-\frac{F\left(z r_{-}, r\right)}{\Delta\left(z, r^{2}\right)}\right\}-\frac{g^{2} F\left(z r_{-}, r\right)}{\pi z(1-z) \Delta\left(z, r^{2}\right)} \\
& =\int_{0}^{1} d y \mathrm{~V}(z, y) \frac{F\left(y r_{-}, r\right)}{\Delta\left(y, r^{2}\right)}-\frac{g^{2} / \pi}{z(1-z)} \frac{F\left(z r_{-}, r\right)}{\Delta\left(z, r^{2}\right)} \quad(\text { if } \quad z \in[0,1]),
\end{aligned}
$$

and outside of this region it is

$$
I_{2}(z, r)=\frac{g^{2}}{\pi} \int_{0}^{1} \frac{d y}{(z-y)^{2}} \frac{F\left(y r_{-}, r\right)}{\Delta\left(y, r^{2}\right)}=\int_{0}^{1} d y \mathrm{~V}_{0}(z, y) \frac{F\left(y r_{-}, r_{-}\right)}{\Delta\left(y, r^{2}\right)} \quad(\text { if } \quad z \notin[0,1]),
$$

where we have defined

$$
\frac{\left(r_{-}\right)^{2}}{\pi} V(p, k) \rightarrow\left\{\begin{array}{ll}
\mathrm{V}(z, y) \equiv \frac{g^{2}}{\pi}\left\{\frac{1}{(z-y)^{2}}-\delta(z-y) \int_{0}^{1} d y^{\prime} \frac{1}{\left(z-y^{\prime}\right)^{2}}\right\} & \text { if } z \in[0,1] \\
\mathrm{V}_{0}(z, y) \equiv \frac{g^{2}}{\pi} \frac{1}{(z-y)^{2}} & \text { if } z \notin[0,1]
\end{array} .\right.
$$

The multiplication by $r_{-}^{2}$ in (A9) is needed to scale the momenta from $k_{-} \rightarrow y$, etc.

We emphasize that the identities (A7) and (A8) hold for both $r_{-}>0$ and $r_{-}<0$. The first case is needed for the bound state equation and the second for electron scattering.

\section{APPENDIX B: IDENTITIES INVOLVING BOUND STATE WAVE FUNCTIONS}

In this appendix we derive a number of identities that hold when $\Phi_{n}(0, r)=\Phi_{n}(1, r)=0$ (i.e. in all cases but the chiral limit). Equation (2.42) leads immediately to the following identity

$$
\int_{0}^{1} d z\left(\frac{m_{1}^{2}}{z}+\frac{m_{2}^{2}}{1-z}\right) \Phi_{n}^{\prime}(z, r) \Phi_{n}(z, r)=0
$$

Integrating by parts gives

$$
m_{1}^{2} \int_{0}^{1} d z \frac{\Phi_{n}^{2}(z, r)}{z^{2}}=m_{2}^{2} \int_{0}^{1} d z \frac{\Phi_{n}^{2}(z, r)}{(1-z)^{2}} .
$$

An additional identity is derived by multiplying (2.42) by $2(1-z) \Phi_{n}^{\prime}(z, r)$ and integrating. First observe that

$$
\begin{aligned}
2 \int_{0}^{1} d z( & 1-z) \Phi_{n}^{\prime}(z, r) f_{0}^{1} d y \frac{\Phi_{n}^{\prime}(y, r)}{(z-y)} \\
& =\int_{0}^{1} \int_{0}^{1} d z d y \Phi_{n}^{\prime}(z, r) \Phi_{n}^{\prime}(y, r) \frac{y-z}{z-y}=0 .
\end{aligned}
$$


Hence, integrating by parts and using the normalization condition (2.40) for the wave function, we obtain

$$
M_{n}^{2}=m_{1}^{2} \int_{0}^{1} d z \frac{\Phi_{n}^{2}(z, r)}{z^{2}}
$$

Similarly we can prove that

$$
M_{n}^{2}=m_{2}^{2} \int_{0}^{1} d z \frac{\Phi_{n}^{2}(z, r)}{(1-z)^{2}}
$$

Additional identities are needed for the study of duality. First, integrate Eq. (2.37) over $z$ to obtain

$$
M_{n}^{2} \int_{0}^{1} d z \Phi_{n}(z, r)=\int_{0}^{1} d z\left(\frac{m_{01}^{2}}{z}+\frac{m_{02}^{2}}{1-z}\right) \Phi_{n}(z, r) .
$$

Next, the commutator of the operator

$$
\mathcal{K} \Phi_{n}(x, r) \equiv f_{0}^{1} d y \frac{\Phi_{n}(y, r)}{y-x}
$$

with the Hamiltonian defined in (2.37) is

$$
[H, \mathcal{K}] \Phi_{n}(x, r)=\int_{0}^{1} d y\left[\frac{m_{01}^{2}}{x y}-\frac{m_{02}^{2}}{(1-x)(1-y)}\right] \Phi_{n}(y, r),
$$

as discussed in Refs. [2, 7]. Multiplying this by $\Phi_{n}(x, r)$ and integtating over $x$ gives zero on the l.h.s., and hence the relation

$$
m_{01}^{2}\left(\int_{0}^{1} d z \frac{\Phi_{n}(z, r)}{z}\right)^{2}=m_{02}^{2}\left(\int_{0}^{1} d z \frac{\Phi_{n}(z, r)}{1-z}\right)^{2} .
$$

Taking the square root of both sides of this equation gives

$$
m_{01} \int_{0}^{1} d z \frac{\Phi_{n}(z, r)}{z}= \pm m_{02} \int_{0}^{1} d z \frac{\Phi_{n}(z, r)}{1-z} \equiv C_{n}
$$

where the sign is positive if the phase of $\Phi_{n}(0, r)$ is the same as $\Phi_{n}(1, r)$ and negative if it is opposite. Since the states are non-degenerate, the phase of the $n$th eigenstate is $(-1)^{(n-1)}$. Combining this with Eq. (B6) gives

$$
M_{n}^{2} \int_{0}^{1} d z \Phi_{n}(z, r)=\left[m_{01}+(-1)^{(n-1)} m_{02}\right] C_{n},
$$

where the constant $C_{n}$ is yet to be determined. In this paper we have chosen the sign of $\Phi_{n}(z, r)$ so that it is always positive as $z \rightarrow 0$, so that $C_{n}>0$ for all $n$.

Our demonstration of duality depends upon the relations

$$
\begin{aligned}
& \lim _{n \rightarrow \infty} C_{n} \rightarrow C_{\infty}=g \sqrt{\pi} \\
& \lim _{n \rightarrow \infty}\left(M_{n+1}^{2}-M_{n}^{2}\right) \rightarrow C_{\infty}^{2}=\pi g^{2},
\end{aligned}
$$


which can be derived approximately using arguments given by 't Hooft [1], and Callan, Coote, and Gross 2], given here for completeness. First, for large $n$, the boundary conditions require that the normalized wave function go like

$$
\Phi_{n}(z, r) \sim \sqrt{2} \sin (n \pi z) .
$$

One test of this approximation is shown in Fig. 13. If $n$ is very large, so that $M_{n} \gg m_{0 i}$, the behavior of the wave function at the endpoints can be ignored, and near $z=1 / 2$ Eq. (2.42) can be approximated

$$
M_{n}^{2} \Phi_{n}(z, r) \simeq-\frac{g^{2}}{\pi} f_{-\infty}^{\infty} \frac{\Phi_{n}^{\prime}(y, r)}{y-z} .
$$

Substituting the ansatz (B14) into this equation gives

$$
\begin{aligned}
M_{n}^{2} \sin (n \pi z) & =-g^{2} n f_{-\infty}^{\infty} \frac{\cos (n \pi y)}{y-z}=-g^{2} n \operatorname{Re} \int_{-\infty}^{\infty} \frac{\cos (n \pi y)}{y-z-i \epsilon} \\
& =\pi g^{2} n \sin (\pi n z) .
\end{aligned}
$$

Hence $M_{n}^{2} \rightarrow \pi g^{2} n$ as $n \rightarrow \infty$ and identity (B13) is proved. To prove (B12) we consider the matrix element of $(\underline{\mathrm{B} 8})$

$$
\begin{aligned}
\int_{0}^{1} \Phi_{n+1}(x, r)[H, \mathcal{K}] \Phi_{n}(x, r) & =2 C_{n+1} C_{n} \\
& =\left(M_{n+1}^{2}-M_{n}^{2}\right) f_{0}^{1} d x d y \frac{\Phi_{n+1}(x, r) \Phi_{n}(y, r)}{y-x} .
\end{aligned}
$$

where we used the fact that the phases of $\Phi_{n}$ and $\Phi_{n+1}$ are opposite. The integral is evaluated in the large $n$ approximation

$$
\begin{aligned}
f_{0}^{1} d x d y \frac{\Phi_{n+1}(x, r) \Phi_{n}(y, r)}{y-x} & \simeq 2 \operatorname{Re} \int_{0}^{1} d x \int_{-\infty}^{\infty} d y \frac{\sin ([n+1] \pi x) \sin (n \pi y)}{y-x-i \epsilon} \\
& =2 \int_{0}^{1} d x \sin ([n+1] \pi x) \cos (n \pi x) \\
& =2\left(1+\frac{1}{2 n+1}\right) \rightarrow 2 .
\end{aligned}
$$

Hence,

$$
C_{n+1} C_{n} \simeq \pi g^{2} \simeq C_{\infty}^{2}
$$

and $(\mathrm{B} 12)$ is proved.

A numerical demonstration of the identities (B10), (B12), and (B13) is given in Figs. 13. The identities are satisfied to better than a few percent for state numbers between 40 and 80 , provided the number of splines is at least 160 (twice the number of the maximum state of interest).

Two additional relations that follow from the completeness relation (2.51) and the bound state equation are needed. If $z \in[0,1]$, then:

$$
\begin{aligned}
& \sum_{n} \Phi_{n}\left(z, r_{n}\right) \int_{0}^{1} d z^{\prime} \Phi_{n}\left(z^{\prime}, r_{n}\right)=1 \\
& \sum_{n}\left(\frac{m_{01}^{2}}{z}+\frac{m_{02}^{2}}{1-z}-M_{n}^{2}\right) \Phi_{n}\left(z, r_{n}\right) \int_{0}^{1} d z^{\prime} \Phi_{n}\left(z^{\prime}, r_{n}\right)=0 .
\end{aligned}
$$



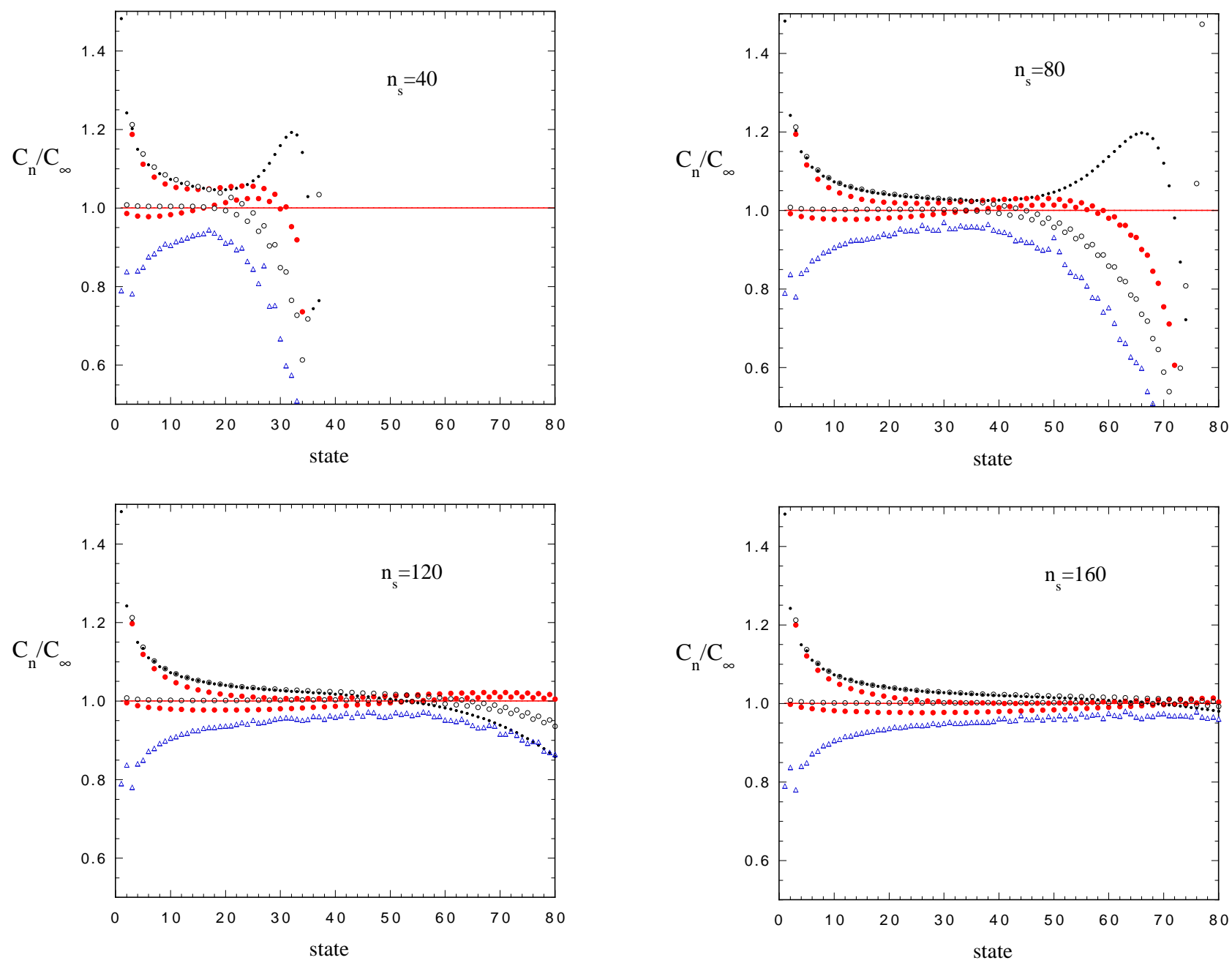

FIG. 13: Numerical test of the relations (B10), (B12), and (B13) for $m_{01}=1.5$ and $m_{02}=3$ and different numbers of splines, $n_{s}$. Solid circles are values of the l.h.s. of (B10) divided by $C_{\infty}$; open circles are the absolute value of the r.h.s. divided by $C_{\infty}$. They should be equal for each state, and approach unity as $n \rightarrow \infty$. The tiny solid squares are the values of $\left(\mu_{n+1}^{2}-\mu_{n}^{2}\right) / \pi^{2}$ which, according (B13), approach unity as $n \rightarrow \infty$. The open triangles are the square of the normalization constant divided by 2 (for wave functions initially normalized with their maximum value equal to unity), and approaches unity if the approximation (B14) is valid.

The proof of the second relation (B21) follows from application of the bound state equation

$$
\begin{aligned}
\sum_{n}( & \left.\frac{m_{01}^{2}}{z}+\frac{m_{02}^{2}}{1-z}-M_{n}^{2}\right) \Phi_{n}\left(z, r_{n}\right) \int_{0}^{1} d z^{\prime} \Phi_{n}\left(z^{\prime}, r_{n}\right) \\
& =\sum_{n} \int_{0}^{1} d y\left[\frac{\Phi_{n}\left(y, r_{n}\right)-\Phi_{n}\left(z, r_{n}\right)}{(y-z)^{2}}\right] \int_{0}^{1} d z^{\prime} \Phi_{n}\left(z^{\prime}, r_{n}\right) \\
& =\sum_{n}\left\{\int_{0}^{z-\epsilon}+\int_{z-\epsilon}^{z+\epsilon}+\int_{z+\epsilon}^{1}\right\} d y\left[\frac{\Phi_{n}\left(y, r_{n}\right)-\Phi_{n}\left(z, r_{n}\right)}{(y-z)^{2}}\right] \int_{0}^{1} d z^{\prime} \Phi_{n}\left(z^{\prime}, r_{n}\right) \\
& \rightarrow \sum_{n} \frac{1}{2} \int_{z-\epsilon}^{z+\epsilon} d y \Phi_{n}^{\prime \prime}\left(z, r_{n}\right) \int_{0}^{1} d z^{\prime} \Phi_{n}\left(z^{\prime}, r_{n}\right)= \\
& =\epsilon \sum_{n} \Phi_{n}^{\prime \prime}\left(z, r_{n}\right) \int_{0}^{1} d z^{\prime} \Phi_{n}\left(z^{\prime}, r_{n}\right) \rightarrow 0
\end{aligned}
$$


where the identity (B20) is used to eliminate the nonsingular integrals over the intervals $[0, z-\epsilon]$ and $[z+\epsilon, 1]$, and the singular part of the integral is proportional to $\epsilon$ which vanishes as $\epsilon \rightarrow 0$.

If Eq. (2.41) is used, modifications of the completeness relation (2.51) and the identity (B20), which hold in the region $z \notin[0,1]$, can be derived. The results are

$$
\begin{aligned}
& \sum_{n}\left(\frac{m_{1}^{2}}{z}+\frac{m_{2}^{2}}{1-z}-M_{n}^{2}\right) \Phi_{n}\left(z, r_{n}\right) \Phi_{n}\left(z^{\prime}, r_{n}\right)=\frac{g^{2} / \pi}{\left(z-z^{\prime}\right)^{2}} \quad z^{\prime} \in[0,1] \\
& \sum_{n}\left(\frac{m_{1}^{2}}{z}+\frac{m_{2}^{2}}{1-z}-M_{n}^{2}\right) \Phi_{n}\left(z, r_{n}\right) \int_{0}^{1} d z^{\prime} \Phi_{n}\left(z^{\prime}, r_{n}\right)=-\frac{g^{2} / \pi}{z(1-z)} .
\end{aligned}
$$

Note that the completeness relation and all of these identities hold for any two quark Hamiltonian. In particular, the Hamiltonian may describe bound states with flavor (where, in general, $m_{1} \neq m_{2}$ ) as well as flavorless bound states where $m_{1}=m_{2}$. In some applications

we will study the coupling of a photon to a $q \bar{q}$ pair. Such a coupling necessarily involves flavorless states only, and we will use the identities for the flavorless states.

\section{APPENDIX C: SUMMATION OF THE QUARK SCATTERING SERIES}

Here we show how to sum this series (2.58) and obtain a useful form for $M$.

Since $V$ is independent of the plus components of momentum, the integrations over the plus components of momentum can be carried out. Using the identity (A7) [with $q_{-}<0$ in this case], the first term on the rhs of (2.58) becomes

$$
\begin{aligned}
\langle M G \mathcal{O}\rangle_{1}\left(p^{\prime} ; q\right) & =i \int \frac{d^{2} p}{\pi^{2}} \frac{V\left(p^{\prime}, p\right) \mathcal{O}\left(p_{-}, q\right)}{d_{2}\left(p_{-}, p_{+}, q\right)} \\
& =\int_{0}^{1} d z \mathrm{~V}\left(z^{\prime}, z\right) \frac{\mathcal{O}\left(p_{-}, q\right)}{\Delta\left(z, q^{2}\right)}-\frac{g^{2}}{\pi z^{\prime}\left(1-z^{\prime}\right)} \frac{\mathcal{O}\left(p_{-}^{\prime}, q\right)}{\Delta\left(z^{\prime}, q^{2}\right)} \\
& =\int_{0}^{1} d z\left\{\mathrm{~V}\left(z^{\prime}, z\right)-\frac{g^{2} \delta\left(z^{\prime}-z\right)}{\pi z(1-z)}\right\} \frac{\mathcal{O}\left(p_{-}, q\right)}{\Delta\left(z, q^{2}\right)} \quad \text { if } z^{\prime} \in[0,1],
\end{aligned}
$$

or

$$
\langle M G \mathcal{O}\rangle_{1}\left(p^{\prime} ; q\right)=i \int \frac{d^{2} p}{\pi^{2}} \frac{V\left(p^{\prime}, p\right) \mathcal{O}\left(p_{-}, q\right)}{d_{2}\left(p_{-}, p_{+}, q\right)}=\int_{0}^{1} d z \mathrm{~V}_{0}\left(z^{\prime}, z\right) \frac{\mathcal{O}\left(p_{-}, q\right)}{\Delta\left(z, q^{2}\right)} \quad \text { if } z^{\prime} \notin[0,1] .
$$

Here the replacement (A9) has been used to convert $V \rightarrow \mathrm{V}$ (or $\mathrm{V}_{0}$ ). Similarly, the second term becomes

$$
\begin{aligned}
\langle M G \mathcal{O}\rangle_{2}\left(p^{\prime} ; q\right)= & i \int \frac{d^{2} k}{\pi^{2}} \frac{V\left(p^{\prime}, k\right)}{d_{2}\left(k_{-}, k_{+}, q\right)} \int_{0}^{1} d z\left\{\mathrm{~V}(y, z)-\frac{g^{2} \delta(y-z)}{\pi z(1-z)}\right\} \frac{\mathcal{O}\left(p_{-}, q\right)}{\Delta\left(z, q^{2}\right)} \\
= & \int_{0}^{1} d y\left\{\mathrm{~V}\left(z^{\prime}, y\right)-\frac{g^{2} \delta\left(z^{\prime}-y\right)}{\pi y(1-y)}\right\} \frac{1}{\Delta\left(y, q^{2}\right)} \\
& \times \int_{0}^{1} d z\left\{\mathrm{~V}(y, z)-\frac{g^{2} \delta(y-z)}{\pi z(1-z)}\right\} \frac{\mathcal{O}\left(p_{-}, q\right)}{\Delta\left(z, q^{2}\right)} \quad \text { if } z^{\prime} \in[0,1],
\end{aligned}
$$


or

$$
\begin{aligned}
&\langle M G \mathcal{O}\rangle_{2}\left(p^{\prime} ; q\right)=\int_{0}^{1} d y \mathrm{~V}_{0}\left(z^{\prime}, y\right) \frac{1}{\Delta\left(y, q^{2}\right)} \\
& \times \int_{0}^{1} d z\left\{\mathrm{~V}(y, z)-\frac{g^{2} \delta(y-z)}{\pi z(1-z)}\right\} \frac{\mathcal{O}\left(p_{-}, q\right)}{\Delta\left(z, q^{2}\right)} \quad \text { if } z^{\prime} \notin[0,1],
\end{aligned}
$$

and the third term follows the same pattern. Replacing $\left(q_{-}\right)^{2} M\left(p^{\prime}, p ; q\right) / \pi \rightarrow \mathrm{M}\left(z^{\prime}, z ; q^{2}\right)$ and $\mathcal{O}\left(p_{-}, q\right) \rightarrow \mathrm{O}\left(z, q^{2}\right)$, the series (2.58), in the interval $z^{\prime} \in[0,1]$, is clearly summed by the following equation

$$
\begin{aligned}
\int_{0}^{1} d z \mathrm{M}\left(z^{\prime}, z ; q^{2}\right) \frac{\mathrm{O}\left(z, q^{2}\right)}{\Delta\left(z, q^{2}\right)}=\int_{0}^{1} d z\left\{\mathrm{~V}\left(z^{\prime}, z\right)-\frac{g^{2} \delta\left(z^{\prime}-z\right)}{\pi z(1-z)}\right\} \frac{\mathrm{O}\left(z, q^{2}\right)}{\Delta\left(z, q^{2}\right)} \\
\quad+\int_{0}^{1} d y \frac{\mathrm{M}\left(z^{\prime}, y ; q^{2}\right)}{\Delta\left(y, q^{2}\right)} \int_{0}^{1} d z\left\{\mathrm{~V}(y, z)-\frac{g^{2} \delta(y-z)}{\pi z(1-z)}\right\} \frac{\mathrm{O}\left(z, q^{2}\right)}{\Delta\left(z, q^{2}\right)} \quad \text { if } z^{\prime} \in[0,1] .
\end{aligned}
$$

Introducing the shorthand notation

$$
F\left(z^{\prime}, z ; q^{2}\right)=\frac{\mathrm{M}\left(z^{\prime}, z ; q^{2}\right)}{\Delta\left(z, q^{2}\right)}
$$

Eq. (C5) can be rearranged into the following form

$$
\begin{gathered}
\int_{0}^{1} d z F\left(z^{\prime}, z ; q^{2}\right)\left\{\left(\Delta\left(z, q^{2}\right)+\frac{g^{2} / \pi}{z(1-z)}\right) \frac{\mathrm{O}\left(z, q^{2}\right)}{\Delta\left(z, q^{2}\right)}-\int_{0}^{1} d y \mathrm{~V}(z, y) \frac{\mathrm{O}\left(y, q^{2}\right)}{\Delta\left(y, q^{2}\right)}\right\} \\
=\int_{0}^{1} d z F\left(z^{\prime}, z ; q^{2}\right)\left(H(z)-q^{2}\right) \frac{\mathrm{O}\left(z, q^{2}\right)}{\Delta\left(z, q^{2}\right)} \\
=\int_{0}^{1} d z\left\{\mathrm{~V}\left(z^{\prime}, z\right)-\frac{g^{2} \delta\left(z^{\prime}-z\right)}{\pi z(1-z)}\right\} \frac{\mathrm{O}\left(z, q^{2}\right)}{\Delta\left(z, q^{2}\right)},
\end{gathered}
$$

which requires that $F$ satisfy the equation

$$
F\left(z^{\prime}, z, q^{2}\right)\left(H(z)-q^{2}\right)=\mathrm{V}\left(z^{\prime}, z\right)-\frac{g^{2} \delta\left(z^{\prime}-z\right)}{\pi z(1-z)} .
$$

This equation is easily solved with the use of the Greens function:

$$
\begin{aligned}
F\left(z^{\prime}, z ; q^{2}\right) & =-\int_{0}^{1} d y\left\{\mathrm{~V}\left(z^{\prime}, y\right)-\frac{g^{2} \delta\left(z^{\prime}-y\right)}{\pi y(1-y)}\right\} G\left(y, z, q^{2}\right) \\
& =-\int_{0}^{1} d y\left\{\mathrm{~V}\left(z^{\prime}, y\right)-\frac{g^{2} \delta\left(z^{\prime}-y\right)}{\pi y(1-y)}\right\} \sum_{n} \frac{\Phi_{n}\left(y, r_{n}\right) \Phi_{n}\left(z, r_{n}\right)}{q^{2}-M_{n}^{2}} \\
& =\sum_{n}\left(M_{n}^{2}-\frac{m_{1}^{2}}{z^{\prime}}-\frac{m_{2}^{2}}{1-z^{\prime}}\right) \frac{\Phi_{n}\left(z^{\prime}, r_{n}\right) \Phi_{n}\left(z, r_{n}\right)}{q^{2}-M_{n}^{2}} .
\end{aligned}
$$

Putting it all together gives Eq. (2.59). 
If $z^{\prime} \notin[0,1]$, the series reduces to the same formal result

$$
\begin{aligned}
\int_{0}^{1} d z \mathrm{M}\left(z^{\prime}, z ; q^{2}\right) \frac{\mathrm{O}\left(z, q^{2}\right)}{\Delta\left(z, q^{2}\right)} & =\int_{0}^{1} d z \mathrm{~V}_{0}\left(z^{\prime}, z\right) \frac{\mathrm{O}\left(z, q^{2}\right)}{\Delta\left(z, q^{2}\right)} \\
& +\int_{0}^{1} d y \mathrm{~V}_{0}\left(z^{\prime}, y\right) \frac{1}{\Delta\left(y, q^{2}\right)} \int_{0}^{1} d z \mathrm{M}\left(y, z ; q^{2}\right) \frac{\mathrm{O}\left(z, q^{2}\right)}{\Delta\left(z, q^{2}\right)} \\
= & \int_{0}^{1} d z \mathrm{~V}_{0}\left(z^{\prime}, z\right) \frac{\mathrm{O}\left(z, q^{2}\right)}{\Delta\left(z, q^{2}\right)}+\sum_{n} \int_{0}^{1} d y \mathrm{~V}_{0}\left(z^{\prime}, y\right) \\
& \times \frac{\Delta\left(y, M_{n}^{2}\right)}{\Delta\left(y, q^{2}\right)} \frac{\Phi_{n}\left(y, r_{n}\right)}{M_{n}^{2}-q^{2}} \int_{0}^{1} d z \Phi_{n}\left(z, r_{n}\right) \mathrm{O}\left(z, q^{2}\right) \\
= & \sum_{n} \Delta\left(z^{\prime}, M_{n}^{2}\right) \frac{\Phi_{n}\left(z^{\prime}, r_{n}\right)}{M_{n}^{2}-q^{2}} \int_{0}^{1} d z \Phi_{n}\left(z, r_{n}\right) \mathrm{O}\left(z, q^{2}\right),
\end{aligned}
$$

where the completeness relation (2.51) and Eq. (2.41) were used in the last step. This shows that (2.59) can be applied for any $z^{\prime} \in[-\infty, \infty]$.

\section{APPENDIX D: SUMMATION OF THE QUARK CURRENT SERIES}

The series (3.7) for the dressed quark current is evaluated in this Appendix. The "first" integral over $k_{+}$in each term in the series is evaluated as in Eq. (C1) or (C2)

$$
\begin{aligned}
i \int \frac{d^{2} k}{(2 \pi)^{2}} V\left(p^{\prime}, k\right) \gamma_{-} S_{i}(k) \gamma^{\mu} S_{i}(k-q) \gamma_{-} \equiv i \int \frac{d^{2} k}{\pi^{2}} \frac{V\left(p^{\prime}, k\right) N_{i}^{\mu}\left(k_{-}, q_{-}\right)}{d_{2}^{i}\left(k_{-}, k_{+}, q\right)} \\
= \begin{cases}\int_{0}^{1} d y\left\{\mathrm{~V}\left(z^{\prime}, y\right)-\frac{g^{2} \delta\left(z^{\prime}-y\right)}{\pi y(1-y)}\right\} \frac{N_{i}^{\mu}\left(k_{-}, q_{-}\right)}{\Delta_{i}\left(y, q^{2}\right)} & \text { if } z^{\prime} \in[0,1] \\
\int_{0}^{1} d y \mathrm{~V}_{0}\left(z^{\prime}, y\right) \frac{N_{i}^{\mu}\left(k_{-}, q_{-}\right)}{\Delta_{i}\left(y, q^{2}\right)} & \text { if } z^{\prime} \notin[0,1],\end{cases}
\end{aligned}
$$

where

$$
N_{i}^{\mu}\left(k_{-}, q_{-}\right)=\frac{\gamma_{-}\left[m_{0 i}+k_{-} \gamma_{+}\right] \gamma^{\mu}\left[m_{0 i}+\left(k_{-}-q_{-}\right) \gamma_{+}\right] \gamma_{-}}{4 k_{-}\left(k_{-}-q_{-}\right)},
$$

and $d_{2}^{i}$ and $\Delta_{i}$ are (A3) and (A6) with equal masses $m_{1}=m_{2}=m_{i}$. Note that the only dependence of the integrand on $k_{+}$was in the denominator $d_{2}^{i}$, so the $k_{+}$integral could be evaluated using the methods of Appendix $\mathbb{C}$ The second integral is similarily evaluated. If $z^{\prime} \in[0,1]$, the following result is obtained for the series Eq. (3.7)

$$
\begin{aligned}
& j_{i}^{\mu}\left(p^{\prime}, p^{\prime}-q\right)=j_{i}^{\mu}\left(z^{\prime}, q^{2}\right)=\gamma^{\mu}+\int_{0}^{1} d y\left\{\mathrm{~V}\left(z^{\prime}, y\right)-\frac{g^{2} \delta\left(z^{\prime}-y\right)}{\pi y(1-y)}\right\} \frac{\mathrm{N}_{i}^{\mu}(y, q)}{\Delta_{i}\left(y^{\prime}, q^{2}\right)} \\
& \quad+\int_{0}^{1} d y^{\prime}\left\{\mathrm{V}\left(z^{\prime}, y^{\prime}\right)-\frac{g^{2} \delta\left(z^{\prime}-y^{\prime}\right)}{\pi y^{\prime}\left(1-y^{\prime}\right)}\right\} \frac{1}{\Delta_{i}\left(y^{\prime}, q^{2}\right)} \int_{0}^{1} d y\left\{\mathrm{~V}\left(y^{\prime}, y\right)-\frac{g^{2} \delta\left(y^{\prime}-y\right)}{\pi y(1-y)}\right\} \frac{\mathrm{N}_{i}^{\mu}(y, q)}{\Delta_{i}\left(y, q^{2}\right)} \\
& +\cdots=\gamma^{\mu}+\int_{0}^{1} d z \mathrm{M}\left(z^{\prime}, y ; q^{2}\right) \frac{\mathrm{N}_{i}^{\mu}(y, q)}{\Delta_{i}\left(y, q^{2}\right)},
\end{aligned}
$$


where $\mathrm{N}_{i}^{\mu}(y, q)=N_{i}^{\mu}\left(k_{-}, q_{-}\right)$, and the series is summed using Eq. (C5) for the scattering matrix M. Using the solution (2.59) for the scattering matrix the result, for all values of $z^{\prime}=p_{-}^{\prime} / q_{-}$, is

$$
j_{i}^{\mu}\left(z^{\prime}, q^{2}\right)=\gamma^{\mu}+\sum_{n} \Delta_{i}\left(z^{\prime}, M_{n}^{2}\right) \frac{\Phi_{n}\left(z^{\prime}, r_{n}\right)}{M_{n}^{2}-q^{2}} \int_{0}^{1} d z \Phi_{n}\left(z, r_{n}\right) \mathrm{N}_{i}^{\mu}(z, q),
$$

where $j_{i}^{\mu}\left(p^{\prime}, p^{\prime}-q\right)=j_{i}^{\mu}\left(z^{\prime}, q^{2}\right)$, and $\Delta_{i}$ is the $\Delta$ of Eq. (A6) with equal masses $m_{1}=m_{2}=m_{i}$.

The same expression also holds for $z^{\prime} \notin[0,1]$, but the derivation differs. As before, the full result can be deduced from the form of the first two terms of the series (3.7). Guided by (D1), and using the steps already displayed in Eq. (C10), we obtain

$$
\begin{aligned}
& j_{i}^{\mu}\left(z^{\prime}, q^{2}\right)=\gamma^{\mu}+\int_{0}^{1} d y \mathrm{~V}_{0}\left(z^{\prime}, y\right) \frac{\mathrm{N}_{i}^{\mu}(y, q)}{\Delta_{i}\left(y^{\prime}, q^{2}\right)} \\
& \quad+\int_{0}^{1} d y^{\prime} \mathrm{V}_{0}\left(z^{\prime}, y^{\prime}\right) \frac{1}{\Delta_{i}\left(y^{\prime}, q^{2}\right)} \int_{0}^{1} d y\left\{\mathrm{~V}\left(y^{\prime}, y\right)-\frac{g^{2} \delta\left(y^{\prime}-y\right)}{\pi y(1-y)}\right\} \frac{\mathrm{N}_{i}^{\mu}(y, q)}{\Delta_{i}\left(y, q^{2}\right)} \\
& \quad+\cdots=\gamma^{\mu}+\int_{0}^{1} d z \mathrm{M}\left(z^{\prime}, y ; q^{2}\right) \frac{\mathrm{N}_{i}^{\mu}(y, q)}{\Delta_{i}\left(y, q^{2}\right)} \\
& \quad=\gamma^{\mu}+\sum_{n} \Delta_{i}\left(z^{\prime}, M_{n}^{2}\right) \frac{\Phi_{n}\left(z^{\prime}, r_{n}\right)}{M_{n}^{2}-q^{2}} \int_{0}^{1} d z \Phi_{n}\left(z, r_{n}\right) \mathrm{N}_{i}^{\mu}(z, q) .
\end{aligned}
$$

We see, as we did with Eq. (2.59), that (D4) holds for all $z^{\prime}$.

Equation (D4) expresses the dressed current as a sum over contributions from the bound states that couple to the photon, showing that vector dominance is a rigorous consequence of this model.

The structure of the numerator $\mathrm{N}_{i}^{\mu}$ depends on the bare current:

$$
\begin{array}{ll}
\gamma_{-}: & \mathrm{N}_{i}^{-}(y, q)=\gamma_{-} \\
\gamma_{+}: & \mathrm{N}_{i}^{+}(y, q)=-\gamma_{-} \frac{m_{0 i}^{2}}{2 q_{-}^{2} y(1-y)} \\
\gamma_{x}: & \mathrm{N}_{i}^{x}(y, q)=-\gamma_{x} \gamma_{-} \frac{m_{0 i}}{2 q_{-} y(1-y)} .
\end{array}
$$

Note the interesting structure of the correction term to the bare current $\gamma_{x}$, which will be of central importance in our discussion below. Substituting these results into (D4) gives the following

$$
\begin{aligned}
j_{i-}\left(z^{\prime}, q^{2}\right) & =\gamma_{-}\left\{1+\sum_{n} \Delta_{i}\left(z^{\prime}, M_{n}^{2}\right) \frac{\Phi_{n}\left(z^{\prime}, r_{n}\right)}{M_{n}^{2}-q^{2}} \int_{0}^{1} d y \Phi_{n}\left(y, r_{n}\right)\right\} \\
j_{i+}\left(z^{\prime}, q^{2}\right) & =\gamma_{+}-\gamma_{-} \frac{m_{0 i}^{2}}{2 q_{-}^{2}}\left\{\sum_{n} \Delta_{i}\left(z^{\prime}, M_{n}^{2}\right) \frac{\Phi_{n}\left(z^{\prime}, r_{n}\right)}{M_{n}^{2}-q^{2}} \int_{0}^{1} d y \frac{\Phi_{n}\left(y, r_{n}\right)}{y(1-y)}\right\} \\
j_{x}\left(z^{\prime}, q^{2}\right) & =\gamma_{x}-\gamma_{x} \gamma_{-} \frac{m_{0 i}}{2 q_{-}}\left\{\sum_{n} \Delta_{i}\left(z^{\prime}, M_{n}^{2}\right) \frac{\Phi_{n}\left(z^{\prime}, r_{n}\right)}{M_{n}^{2}-q^{2}} \int_{0}^{1} d y \frac{\Phi_{n}\left(y, r_{n}\right)}{y(1-y)}\right\} .
\end{aligned}
$$

We organize these expressions by introducing the quark form factor, defined in Eq. (3.8). This immediately gives Eq. (3.9) for the $j_{-}$current. The other currents in (D7) can also 
be written in terms of $F_{i}$. To this end note that the bound state equation for equal mass quarks implies that

$$
M_{n}^{2} \int_{0}^{1} d y \Phi_{n}\left(y, r_{n}\right)=m_{0 i}^{2} \int_{0}^{1} d y \frac{\Phi_{n}\left(y, r_{n}\right)}{y(1-y)} .
$$

Hence the quark mass can be removed from the second term in $j_{+}$, giving

$$
\begin{aligned}
g_{i}\left(z^{\prime}, q\right) & \equiv-\frac{m_{0 i}^{2}}{2 q_{-}^{2}}\left\{\sum_{n} \Delta_{i}\left(z^{\prime}, M_{n}^{2}\right) \frac{\Phi_{n}\left(z^{\prime}, r_{n}\right)}{M_{n}^{2}-q^{2}} \int_{0}^{1} d y \frac{\Phi_{n}\left(y, r_{n}\right)}{y(1-y)}\right\} \\
& =\frac{q_{+}}{q_{-}}\left\{\sum_{n} \frac{M_{n}^{2}}{Q^{2}} \Delta_{i}\left(z^{\prime}, M_{n}^{2}\right) \frac{\Phi_{n}\left(z^{\prime}, r_{n}\right)}{M_{n}^{2}+Q^{2}} \int_{0}^{1} d y \Phi_{n}\left(y, r_{n}\right)\right\} \\
& =-\frac{q_{+}}{q_{-}} F_{i}\left(z^{\prime}, Q^{2}\right)+\frac{q_{+}}{q_{-} Q^{2}}\left\{\sum_{n} \Delta_{i}\left(z^{\prime}, M_{n}^{2}\right) \Phi_{n}\left(z^{\prime}, r_{n}\right) \int_{0}^{1} d y \Phi_{n}\left(y, r_{n}\right)\right\}
\end{aligned}
$$

where $g_{i}\left(z^{\prime}, q\right)$ defined by this equation should not be confused with the two body Greens function or the bound state vertex function. If $z^{\prime} \in[0,1]$, the second term can be reduced using the identities (B20) and (B21)

$$
\sum_{n}\left[\frac{m_{0 i}^{2}}{z^{\prime}\left(1-z^{\prime}\right)}-M_{n}^{2}-\frac{g^{2} / \pi}{z^{\prime}\left(1-z^{\prime}\right)}\right] \Phi_{n}\left(z^{\prime}, r_{n}\right) \int_{0}^{1} d y \Phi_{n}\left(y, r_{n}\right)=-\frac{g^{2}}{\pi} \frac{1}{z^{\prime}\left(1-z^{\prime}\right)} .
$$

The same result is obtained directly from identity (B24) if $z^{\prime} \notin[0,1]$. Hence, for all $z^{\prime}$,

$$
g_{i}\left(z^{\prime}, q\right)=-\frac{q_{+}}{q_{-}} F_{i}\left(z^{\prime}, Q^{2}\right)+\frac{g^{2}}{2 \pi} \frac{1}{q_{-}^{2} z^{\prime}\left(1-z^{\prime}\right)}=-\frac{q_{+}}{q_{-}} F_{i}\left(z^{\prime}, Q^{2}\right)-\frac{g^{2}}{2 \pi} \frac{1}{p_{-}^{\prime} p_{-}} .
$$

Finally, the $j_{x}$ component of the current can be similarly reduced. The second term in $j_{x}$ is proportional to

$$
\begin{aligned}
H_{i}\left(z^{\prime}, q\right) & \equiv-\frac{m_{0 i}}{2 q_{-}}\left\{\sum_{n} \Delta_{i}\left(z^{\prime}, M_{n}^{2}\right) \frac{\Phi_{n}\left(z^{\prime}, r_{n}\right)}{M_{n}^{2}-q^{2}} \int_{0}^{1} d y \frac{\Phi_{n}\left(y, r_{n}\right)}{y(1-y)}\right\} \\
& =-\frac{1}{2 q_{-} m_{0 i}}\left\{\sum_{n} M_{n}^{2} \Delta_{i}\left(z^{\prime}, M_{n}^{2}\right) \frac{\Phi_{n}\left(z^{\prime}, r_{n}\right)}{M_{n}^{2}+Q^{2}} \int_{0}^{1} d y \Phi_{n}\left(y, r_{n}\right)\right\} \\
& =-\frac{q_{+}}{m_{0 i}} F_{i}\left(z^{\prime}, Q^{2}\right)-\frac{}{2 q_{-} m_{0 i}}\left\{\sum_{n} \Delta_{i}\left(z^{\prime}, M_{n}^{2}\right) \Phi_{n}\left(z^{\prime}, r_{n}\right) \int_{0}^{1} d y \Phi_{n}\left(y, r_{n}\right)\right\} .
\end{aligned}
$$

The part of the second term in \{\} is the same constant derived above, and combining the results gives Eq. (3.11).

\section{APPENDIX E: KINEMATICS FOR DIS}

Kinematics for DIS in the c.m. frame are given in this Appendix. When $Q^{2}$ is very large, the components of $P$ and $q$ in this frame are (see Ref. [14])

$$
q_{+}=Q \sqrt{\frac{1-x}{2 x}}\left[1-\frac{M_{0}^{2} x(1-2 x)}{2 Q^{2}(1-x)}+\ldots\right]
$$




$$
\begin{aligned}
& q_{-}=-Q \sqrt{\frac{x}{2(1-x)}}\left[1+\frac{M_{0}^{2} x(1-2 x)}{2 Q^{2}(1-x)}+\ldots\right] \\
& P_{+}=\frac{M_{0}^{2}}{Q} \sqrt{\frac{x(1-x)}{2}}\left[1-\frac{M_{0}^{2} x\left(1-2 x^{2}\right)}{2 Q^{2}(1-x)}+\ldots\right] \\
& P_{-}=\frac{Q}{\sqrt{2 x(1-x)}}\left[1+\frac{M_{0}^{2} x\left(1-2 x^{2}\right)}{2 Q^{2}(1-x)}+\ldots\right] .
\end{aligned}
$$

The energy and momentum of particles 1 and 2 in the final state are

$$
\begin{aligned}
& p_{z}= \pm \frac{Q}{2} \sqrt{\frac{1-x}{x}}\left[1+\frac{x\left(M_{0}^{2}-2 m_{1}^{2}-2 m_{2}^{2}\right)}{2 Q^{2}(1-x)}+\cdots\right] \\
& p_{10}=\sqrt{m_{1}^{2}+p_{z}^{2}}=\frac{Q}{2} \sqrt{\frac{1-x}{x}}\left[1+\frac{x\left(M_{0}^{2}+2 m_{1}^{2}-2 m_{2}^{2}\right)}{2 Q^{2}(1-x)}+\cdots\right] \\
& p_{20}=\sqrt{m_{2}^{2}+p_{z}^{2}}=\frac{Q}{2} \sqrt{\frac{1-x}{x}}\left[1+\frac{x\left(M_{0}^{2}-2 m_{1}^{2}+2 m_{2}^{2}\right)}{2 Q^{2}(1-x)}+\cdots\right] .
\end{aligned}
$$

There are two possibilities corresponding to the two terms in the current (4.10). The first term in the current will be large only if the momentum of particle 1 is in the direction of $q_{3}$ (i.e. $p_{1 z}=\left|p_{z}\right|>0$ ). [Choosing it in the opposite direction requires a very large momentum flow through the wave function, and is suppressed.] In this case

$$
\begin{aligned}
& p_{1+}^{(1)}=Q \sqrt{\frac{1-x}{2 x}}\left[1+\frac{x\left(M_{0}^{2}-2 m_{2}^{2}\right)}{2 Q^{2}(1-x)}+\cdots\right] \\
& p_{1-}^{(1)}=\sqrt{\frac{x}{2(1-x)}} \frac{m_{1}^{2}}{Q}\left[1-\frac{x\left(M_{0}^{2}-2 m_{2}^{2}\right)}{2 Q^{2}(1-x)}+\cdots\right] \\
& p_{2+}^{(1)}=\sqrt{\frac{x}{2(1-x)}} \frac{m_{2}^{2}}{Q}\left[1-\frac{x\left(M_{0}^{2}-2 m_{1}^{2}\right)}{2 Q^{2}(1-x)}+\cdots\right] \\
& p_{2-}^{(1)}=Q \sqrt{\frac{1-x}{2 x}}\left[1+\frac{x\left(M_{0}^{2}-2 m_{1}^{2}\right)}{2 Q^{2}(1-x)}+\cdots\right],
\end{aligned}
$$

where the superscript (1) is a reminder that these relations hold only for the first term in the current. The momentum relations for the second term in the current (4.10) follow by interchanging 1 and 2 on both sides of these equations. We see that the first term in the current gives $p_{1+} \rightarrow Q$ and $p_{2+} \rightarrow 1 / Q$, while the second term gives $p_{1+} \rightarrow 1 / Q$ and $p_{2+} \rightarrow Q$. The two terms describe kinematically distinct regions of phase space, and their interference can safely be neglected when the total cross section is computed. Using the definitions (4.7) and (4.9), $z$ and $z^{\prime}$ can be easily related to the Bjorken variable $x$. We have

$$
\begin{gathered}
z=1-\frac{p_{2-}^{(1)}}{P_{-}} \simeq 1-(1-x)=x \\
z^{\prime}=1-\frac{p_{1-}^{(2)}}{P_{-}} \simeq 1-(1-x)=x
\end{gathered}
$$




\section{APPENDIX F: THE BOUND STATE TRANSITION CURRENTS}

This Appendix includes details of the evaluation of the transition currents shown in Fig. 10. The $e_{1}$ term will be evaluated first. The $e_{2}$ term can then be obtained by a simple substitution, as discussed at the end of this Appendix.

The $e_{1}$ term, given in Eq. (3.13), is reduced by introducing the momentum fractions

$$
\xi=\frac{(P+k)_{-}}{P_{-}} \quad \eta=\frac{\left(P_{f}+k\right)_{-}}{q_{-}} \quad y=-\frac{q_{-}}{P_{-}},
$$

so that

$$
\frac{P_{f-}}{P_{-}}=1-y \quad \frac{(P+k)_{-}}{q_{-}}=\eta-1 \quad \xi^{\prime}=\frac{\left(P_{f}+k\right)_{-}}{P_{f-}}=\frac{\xi-y}{1-y} .
$$

In this notation the $x$-type transverse quark current, evaluated in Eq. (3.11), is

$$
\begin{aligned}
j_{1}^{x} & =\gamma_{x}-\gamma_{x} \gamma_{-}\left\{\frac{g^{2}}{2 \pi m_{01}} \frac{q_{-}}{\left(P_{f-}+k_{-}\right)\left(P_{-}+k_{-}\right)}-\frac{q_{+}}{m_{01}} F_{1}\left(\eta, Q^{2}\right)\right\} \\
& =\gamma_{x}+\gamma_{x} \gamma_{-} H_{1}(\eta, q)
\end{aligned}
$$

First evaluate the numerators, $N^{\mu}$, of the traces in (3.13). The numerator $N_{x}$ is

$$
\begin{aligned}
N_{x}= & \operatorname{Tr}\left[\gamma_{-} \gamma_{x}\left\{m_{01}+\left(P_{f}+k\right)_{-} \gamma_{+}\right\}\left(\gamma_{x}+\gamma_{x} \gamma_{-} H_{1}(\eta, q)\right)\right. \\
& \left.\times\left\{m_{01}+(P+k)_{-} \gamma_{+}\right\} \gamma_{-}\left(k_{-} \gamma_{+}\right)\right] \\
= & 16 k_{-}\left[m_{01} q_{-}+2\left(P_{f}+k\right)_{-}(P+k)_{-} H_{1}(\eta, q)\right] \\
= & 16 k_{-} q_{-} \frac{m_{1}^{2}}{m_{01}}-32\left(P_{f}+k\right)_{-}(P+k)_{-} \frac{q_{+}}{m_{01}} F_{1}\left(\eta, Q^{2}\right) \\
= & \frac{16 k_{-} q_{-}}{m_{01}}\left(m_{1}^{2}-\eta(1-\eta) Q^{2} F_{1}\left(\eta, Q^{2}\right)\right)
\end{aligned}
$$

where the trace has been evaluated in a Dirac space of four dimensions. The numerator of the minus component of the current has only one term

$$
\begin{aligned}
N_{-} & =\operatorname{Tr}\left[\gamma_{-}\left(P_{f}+k\right)_{-} \gamma_{+} \gamma_{-}(P+k)_{-} \gamma_{+} \gamma_{-}\left(k_{-} \gamma_{+}\right)\right]\left[1+F_{1}\left(\eta, Q^{2}\right)\right] \\
& =32 k_{-}\left(P_{f}+k\right)_{-}(P+k)_{-}\left[1+F_{1}\left(\eta, Q^{2}\right)\right] \\
& =16 k_{-} \frac{q_{-}}{q_{+}} \eta(1-\eta) Q^{2}\left[1+F_{1}\left(\eta, Q^{2}\right)\right] .
\end{aligned}
$$

The trace for the plus component is

$$
\begin{aligned}
N_{+}= & \operatorname{Tr}\left[\gamma_{-}\left\{m_{01}+\left(P_{f}+k\right)_{-} \gamma_{+}\right\}\left(\gamma_{+}+\gamma_{-} G_{1}(\eta, q)\right)\right. \\
& \left.\times\left\{m_{01}+(P+k)_{-} \gamma_{+}\right\} \gamma_{-}\left(k_{-} \gamma_{+}\right)\right] \\
= & 16 k_{-} m_{1}^{2}-32 k_{-}\left(P_{f}+k\right)_{-}(P+k)_{-} \frac{q_{+}}{q_{-}} F_{1}\left(\eta, Q^{2}\right) \\
= & 16 k_{-}\left(m_{1}^{2}-\eta(1-\eta) Q^{2} F_{1}\left(\eta, Q^{2}\right)\right) .
\end{aligned}
$$


Inserting the numerator (F5) into the general result (3.13) gives the following result for the minus component of the current

$$
\begin{aligned}
& \left.\left\langle f_{-}\left|\mathcal{J}_{-}\left(P_{f}, P\right)\right| i_{-}\right\rangle\right|_{e_{1} \text { term }} \\
& =32 i e_{1} \int \frac{d^{2} k}{(2 \pi)^{2}} \frac{\left(P_{f}+k\right)_{-}(P+k)_{-} k_{-} G_{f}\left(-P_{f}-k,-k\right) G_{i}(P+k, k)}{\left[m_{1}^{2}-\left(P_{f}+k\right)^{2}-i \epsilon\right]\left[m_{1}^{2}-(P+k)^{2}-i \epsilon\right]\left[m_{2}^{2}-k^{2}-i \epsilon\right]}\left[1+F_{1}\left(\eta, Q^{2}\right)\right] \\
& =32 i e_{1} \int \frac{d^{2} k}{(2 \pi)^{2}} \frac{1}{d_{3}} G_{f}\left(-P_{f}-k,-k\right) G_{i}(P+k, k)\left[1+F_{1}\left(\eta, Q^{2}\right)\right]
\end{aligned}
$$

where the subscript - on $f_{-}$reminds us that the final state must have a pure $\gamma_{-}$structure (if the ground state does), $d_{3}$ is the product of the denominators of the three quark propagators, with three poles in $k_{+}$

$d_{3}=\left[\frac{m_{1}^{2}}{\xi^{\prime} P_{f-}}-\frac{M_{f}^{2}}{P_{f-}}-2 k_{+}-i \epsilon_{a}\right]\left[\frac{m_{1}^{2}}{\xi P_{-}}-\frac{M_{i}^{2}}{P_{-}}-2 k_{+}-i \epsilon_{b}\right]\left[\frac{m_{2}^{2}}{(\xi-1) P_{-}}-2 k_{+}-i \epsilon_{c}\right]$,

and the $\epsilon$ 's change sign according to

$$
\begin{aligned}
\epsilon_{a} & =\epsilon /\left(P_{f}+k\right)_{-}=\epsilon /\left(\xi^{\prime} P_{f-}\right)=\epsilon /\left[(\xi-y) P_{-}\right] \\
\epsilon_{b} & =\epsilon /(P+k)_{-}=\epsilon /\left(\xi P_{-}\right) \\
\epsilon_{c} & =\epsilon / k_{-}=\epsilon /\left[(\xi-1) P_{-}\right] .
\end{aligned}
$$

Since the vertex functions $G$ do not depend on $k_{+}$, we can evaluate the $k_{+}$integral. It will be nonzero only when the three poles of $(\mathrm{F} 8)$ do not all lie in the same half plane. Since $q_{-}<0$ for electron scattering, $P_{-}>P_{f_{-}}>0$, which implies that

$$
0<y<1
$$

Hence

$$
\xi-1<\xi-y<\xi
$$

and all three poles will be in the same half of the complex plane (giving zero for the integral) unless

$$
0<\xi<1
$$

There are two terms, depending on the sign of $\xi-y$. Closing the $k_{+}$contour in the upper half plane, and using $d k_{-}=P_{-} d \xi$ and $(\xi-1) P_{-}=\left(\xi^{\prime}-1\right) P_{f-\text { gives }}$

$$
\begin{aligned}
\left.\left\langle f_{-}\left|\mathcal{J}_{-}\left(P_{f}, P\right)\right| i_{-}\right\rangle\right|_{e_{1}}= & 16 e_{1} P_{-}^{2} P_{f-} \int_{0}^{1} \frac{d \xi}{(2 \pi)} \frac{G_{f}\left(\xi^{\prime}, P_{f}\right) G_{i}(\xi, P)}{\Delta\left(\xi^{\prime}, P_{f}^{2}\right) \Delta\left(\xi, P^{2}\right)}\left[1+F_{1}\left(\eta, Q^{2}\right)\right] \\
& -16 e_{1} P_{-}^{2} P_{f-} \int_{0}^{y} \frac{d \xi}{(2 \pi)} \frac{G_{f}\left(\xi^{\prime}, P_{f}\right) G_{i}(\xi, P)\left[1+F_{1}\left(\eta, Q^{2}\right)\right]}{\Delta\left(\xi^{\prime}, P_{f}^{2}\right)\left(\Delta\left(\xi, P^{2}\right)-\frac{\Delta\left(\xi^{\prime}, P_{f}^{2}\right)}{(1-y)}\right)}
\end{aligned}
$$

where the first term is the contribution from the pole due to the zero in the third term in (F8) above, and the second from the pole due to the zero in the first term. Using the 
definition (2.39) of the wave function and combining the two contributions in the region $[0, y]$ we get Eq. (3.14) with

$$
\begin{aligned}
\mathcal{R} & =\frac{\Delta\left(\xi^{\prime}, P_{f}^{2}\right)}{\Delta\left(\xi^{\prime}, P_{f}^{2}\right)-(1-y) \Delta\left(\xi, P^{2}\right)} \\
& =\frac{\xi\left[\xi^{\prime}\left(1-\xi^{\prime}\right) M_{f}^{2}-\left(1-\xi^{\prime}\right) m_{1}^{2}-\xi^{\prime} m_{2}^{2}\right]}{\left(1-\xi^{\prime}\right)\left\{\xi \xi^{\prime}\left(M_{f}^{2}-(1-y) M_{i}^{2}\right)-y m_{1}^{2}\right\}} \rightarrow 1 \quad \text { if } \quad M_{f}^{2} \rightarrow \infty .
\end{aligned}
$$

This factor is needed for positive values of $\xi$ in the interval $[0, y]$ and negative values of $\xi^{\prime}$ in the interval $[-y /(1-y), 0]$. In this region the denominator has a zero only if $m_{1}^{2}<0$. In order to avoid the discussion of such cases we limit numerical applications to cases with $m_{1}^{2}>0$.

Results for the other components of the transition current, Eq. (3.16), are obtained by a similar arguement using ( $(\underline{\mathrm{F} 4})$ and $(\underline{\mathrm{F} 6})$ in place of ([F5).

Now consider the modifications required in order to evaluate the $e_{2}$ term. Using the momenta defined in Fig. 10, and the same definitions of momentum fractions (F1) and (F2), the $e_{2}$ term is obtained from the $e_{1}$ term simply by substituting $m_{1} \leftrightarrow m_{2}$ and $e_{1} \rightarrow e_{2}$. However, the momentum fractions in the wave functions are, by convention, the fraction of the momentum carried by the quark $m_{1}$, and the momentum fraction in the quark form factor is that of the the outgoing quark, and hence these functions must be written in terms of

$$
\begin{aligned}
\xi_{2} & =\frac{-k_{-}}{P_{-}}=1-\xi \\
\xi_{2}^{\prime} & =\frac{-k_{-}}{P_{f-}}=1-\xi^{\prime} \\
\eta_{2} & =\frac{-(P+k)_{-}}{q_{-}}=1-\eta,
\end{aligned}
$$

These observations lead immediately to the final result (3.17).

\section{APPENDIX G: THE MODIFIED CUBIC SPLINES}

The two body equations were solved using a modification of the standard cubic splines employed previously in many problems. The standard splines are defined on 4 segments of length $h$, bounded by the 5 points $a=(n-2) h, b=a+h, c=b+h, d=c+h, e=d+h$ :

$$
S_{n}(x)=\frac{1}{4} \begin{cases}\frac{(x-a)^{3}}{h^{3}} & \text { if } a<x<b \\ 1+3\left[\frac{(x-b)}{h}+\frac{(x-b)^{2}}{h^{2}}-\frac{(x-b)^{3}}{h^{3}}\right] & \text { if } b<x<c \\ 1+3\left[\frac{(d-x)}{h}+\frac{(d-x)^{2}}{h^{2}}-\frac{(d-x)^{3}}{h^{3}}\right] & \text { if } c<x<d \\ \frac{(e-x)^{3}}{h^{3}} & \text { if } d<x<e .\end{cases}
$$

If the interval $[0,1]$ is spanned by $n_{s}-2$ standard splines, there must be $n_{s}+1$ segments, of length $h=1 /\left(n_{s}+1\right)$. The first spline is numbered $n=2$ beginning at $x=0$ and the last is numbered $n_{s}-1$ ending at $x=1$. 

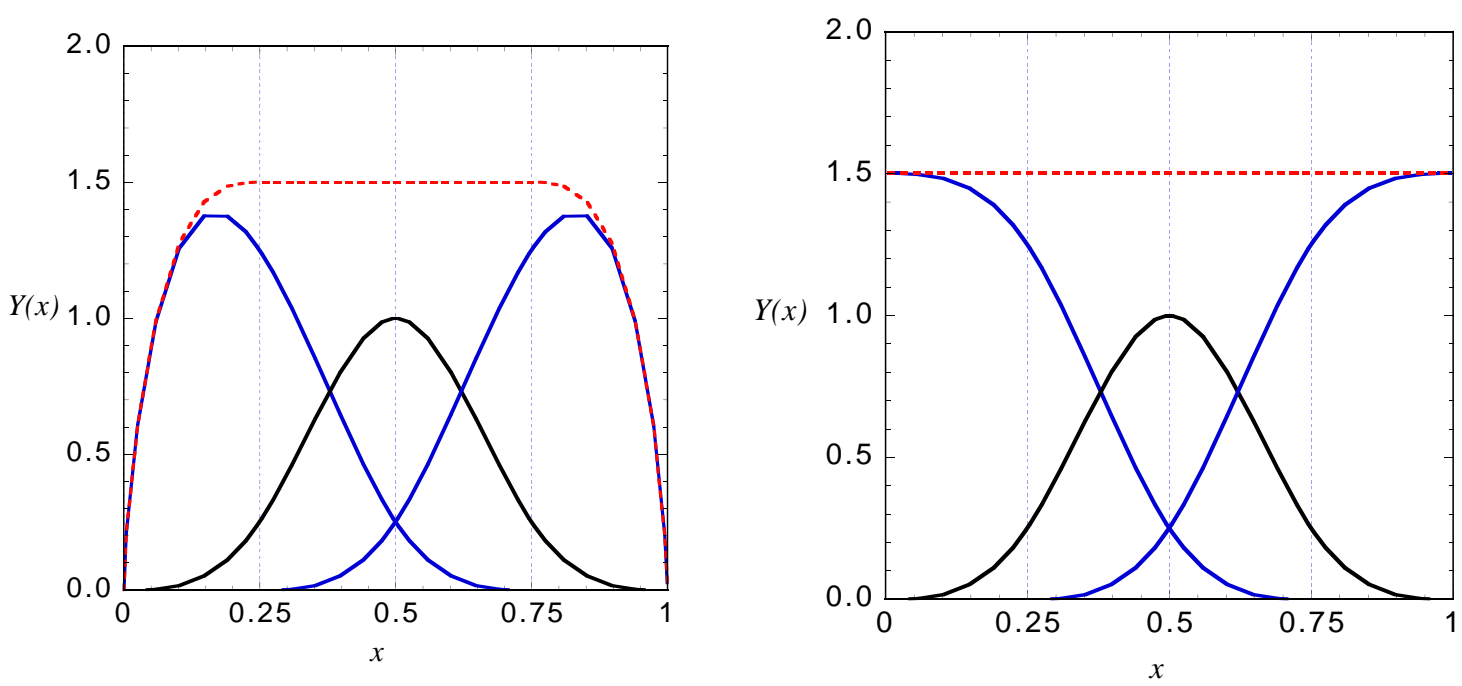

FIG. 14: The modified cubic splines for the minimal case of $n_{s}=3$, with four segments. In the left figure the left- and right-most splines at the boundary approach 0 as $x^{0.6705}$ and $(1-x)^{0.6705}$ appropriate to quark masses $m_{01}=m_{02}=1.5$. In the right figure the bare quark masses are zero and the splines approach a constant at the boundaries. Note that the sum of the splines (the dotted line) is a constant in the central region, and throughout the whole region in the chiral case.

The modified splines used in this paper consist of the standard splines plus one additional, non-standard spline inserted at the beginning and end of the interval $[0,1]$. These nonstandard splines are defined on only three segments, and will be numbered 1 and $n_{s}$. They are

$$
\begin{aligned}
& S_{1}(x)= \begin{cases}d_{1} \frac{x^{\beta_{1}}}{h_{1}}+d_{2} \frac{x^{2}}{h^{2}}+d_{3} \frac{x^{3}}{h^{3}} & \text { if } 0<x<h \\
\frac{1}{4}+\frac{3}{4}\left[\frac{(2 h-x)}{h}+\frac{(2 h-x)^{2}}{h^{2}}-\frac{(2 h-x)^{3}}{h^{3}}\right] & \text { if } h<x<2 h \\
\frac{(3 h-x)^{3}}{4 h^{3}} & \text { if } 2 h<x<3 h\end{cases} \\
& S_{n_{s}}(x)= \begin{cases}\frac{(x-1+3 h)^{3}}{4 h^{3}} & \text { if } 1-3 h<x<1-2 h \\
\frac{1}{4}+\frac{3}{4}\left[\frac{(1-h-x)}{h}+\frac{(1-h-x)^{2}}{h^{2}}-\frac{(1-h-x)^{3}}{h^{3}}\right] & \text { if } 1-2 h<x<1-h \\
d_{1} \frac{(1-x)^{\beta_{2}}}{h^{\beta_{2}}}+d_{2} \frac{(1-x)^{2}}{h^{2}}+d_{3} \frac{(1-x)^{3}}{h^{3}} & \text { if } 1-h<x<1 .\end{cases}
\end{aligned}
$$

where the $\beta_{i}$ are the fractional exponents determined by the boundary conditions [given in Eq. [2.49)], and the coefficients $d_{i}$ depend on the exponents $\beta$

$$
d_{1}=\frac{3}{(3-\beta)(2-\beta)}, \quad d_{2}=\frac{3(1-\beta)}{(2-\beta)}, \quad d_{3}=\frac{(2 \beta-3)}{(3-\beta)},
$$

where $\beta=\beta_{1}$ for $S_{1}$ or $\beta_{2}$ for $S_{n_{s}}$. These coefficients were fixed by the requirement that the spline and its first two derivatives be continuous. Figure 14 shows two examples of the modified splines for the minimal number $n_{s}=3$. In this case the central spline spans the full interval $[0,1]$ and has 4 segments.

Using these splines as a basis, the equation is reduced to a matrix equation that is solved with the standard eigenvalue subroutine packages. 
[1] G. 't Hooft, Nucl. Phys. B75, 461 (1974).

[2] C. G. Callan, N. Coote and D. J. Gross, Phys. Rev. D 13, 1649 (1976).

[3] M. Einhorn, Phys. Rev. D 14, 3451 (1976).

[4] M. I Bars, M. B. Green, Phys. Rev D 15, (1978).

[5] C. G. Callan and D. J. Gross, Phys. Rev. Lett. 22, 156 (1969).

[6] M. Burkardt, Phys. Rev. D 62, 094003 (2000) arXiv:hep-ph/0005209.

[7] M. Burkardt and N. Uraltsev, Phys. Rev. D 63, 014004 (2001) arXiv:hep-ph/0005278.

[8] V. Schon and M. Thies, Contribution to the Festschrift in honor of Boris Ioffe, M. Shifman, (ed). In At the frontier of particle physics, vol. 3 p. 1945 (2000) arXiv:hep-th/0008175.

[9] M. Burkardt, F. Lenz and M. Thies, Phys. Rev. D 65, 125002 (2002) arXiv:hep-th/0201235.

[10] Y. S. Kalashnikova and A. V. Nefediev, Phys. Usp. 45, 347 (2002) [Usp. Fiz. Nauk 172, 378 (2002)] arXiv:hep-ph/0111225.

[11] B. Grinstein, R. F. Lebed, Phys. Rev. D 57:1366 (1998) hep-ph/9708396.

[12] R. F. Lebed, Talk given at 3rd International Conference in Quark Confinement and Hadron Spectrum (Confinement III), Newport News, VA, 7-12 Jun 1998; Published in Newport News 1998, Quark confinement and the hadron spectrum III, p.161 (1998) hep-ph/9808452.

[13] R. F. Lebed, N. G. Uraltsev, Phys. Rev. D 62:094011 (2000) hep-ph/0006346.

[14] Z. Batiz and F. Gross, Phys. Rev. C 58, 2963 (1998).

Note that the square bracket in the second of Eqs. (5.8) should be $\left[1-\frac{M^{2} x(1-2 x)^{2}}{2 Q^{2}(1-x)}\right]$ instead of $\left[-\frac{M^{2} x(1-2 x)^{2}}{2 Q^{2}(1-x)}\right]$.

[15] Z. Batiz and F. Gross, in preparation.

[16] F. Gross and J. Milana, Phys. Rev. D 43(1991) 2401.

F. Gross and J. Milana, Phys. Rev. D 45 (1992) 969.

F. Gross and J. Milana, Phys. Rev. D 50 (1994) 3332.

[17] C. Savkli and F. Gross, Phys. Rev. C 63, 035208 (2001).

[18] F. Gross and D. O. Riska, Phys. Rev. C 36, 1928 (1987).

[19] F. E. Close, N. Isgur, Phys. Lett. B 509, 81 (2001) hep-ph/0102067.

[20] N. Isgur, S. Jeschonnek, W. Melnitchouk and J. W. Van Orden, Phys. Rev. D 64:054005 (2001). 